\title{
PROTECTOR CONTROL: EXTENSION TO A CLASS OF NONLINEAR DISTRIBUTED SYSTEMS
}

\author{
Youssef QARAAI, ABDES SAMED BERNOUSSI \\ MPPRN Laboratory \\ Faculty of Sciences and Techniques, B.P. 416, Tangier, Morocco \\ e-mail: \{qaraai_youssef, bersamed16\}@yahoo.fr
}

\begin{abstract}
We present an extension of the protector control scheme introduced for the linear case in a previous work to a class of nonlinear systems. The systems considered are assumed to have a finite propagation velocity while the initial state is subject to a spreading disturbance. We characterize such a control first by using the remediability approach to the resulting nonlinear delay system, and then by coupling families of transformations and the delay approach. To illustrate this work, we provide a simulation example.
\end{abstract}

Keywords: nonlinear distributed systems, disturbance, spreadability, vulnerability, remediability, protector control.

\section{Introduction}

In environmental modeling, numerous studies have shown that transport systems describe many natural phenomena (Beltrami, 1987; Diaz and Lions, 1993). Various situations have been considered according to the initial and boundary conditions (Dautray and Lions, 1984). Generally, it concerns the velocity field and a portion (or all) of the boundary of the geometrical domain on which the system evolves. In this work, we consider a nonlinear system which propagates with a finite velocity and its initial condition is subject to a disturbance. Thus, where the disturbance is assumed to be spreadable, we will see how to protect certain areas which are vulnerable to the effects of such a disturbance. This is an extension of the protector control issue to this class of nonlinear systems. We explore this concept based on several concepts of regional analysis.

Indeed, regarding the important role of the space variable in the analysis and control of distributed systems, regional analysis of such systems is limited to the study of their behavior just on a subset of the global domain (El Jai et al., 1995; El Jai (2002; 2004)). For systems undergoing an external disturbance, the problem of control arises. This leads (Afifi et al. $(2000 ; 2001)$ ) to the study of the sources and their regional detection when they are unknown. The concept of the spy sensor is introduced to reconstruct these sources.

In fact, knowing this disturbance, another problem consists in describing its spatial evolution over a time interval. For that purpose, we use the concept of spreadability (El Jai and Kassara, 1994; 1996; Bernoussi and El Jai, 2000; Bernoussi et al., 2001). Hence, this leads to the problem of the influence of this disturbance on some given areas, and also the possibility of subjecting them to controls to achieve some specified objectives. Indeed, given a fixed zone in the global domain on which the system evolves, this zone is called vulnerable if it is likely to be reached (infected) by a given property (Bernoussi and Amharref, 2003; Bernoussi, 2007). Moreover, if this zone is reached, then the problem of determining a control which makes it possible to compensate the disturbance effects on such a zone at a certain time is regional remediability. This problem has been widely studied in the case of linear systems (Afifi et al., 2002).

As it is not always possible to remedy some disturbances on a given area such as incurable epidemics, or the remediable control exists but with a very high cost, we think about the possibility of protecting such a zone from being reached (or contaminated). This enabled us to introduce the concept of the protector control in the case of linear systems (Qaraai et al., 2006; Bernoussi , 2010). The principle is to be able to prevent such effects from reaching the area during all the time interval on which the linear system evolves.

To characterize the solutions of the protector control problem, we used two different approaches: the first one 
concerns the so-called adaptable families of transformations used to characterize the spreadability of the system considered (Bernoussi and El Jai, 2000), while the other is based on regional remediability not for the original system but for some suitable delay system. A common aspect of these two approaches is to deviate the property generated by the disturbance effects for not passing into a given vulnerable zone.

On the other hand, and given the fact that the phenomena that motivate us to explore the concept of the protector control are modeled by nonlinear systems, in this work we will extend the protector control concept introduced for the linear case (Bernoussi , 2010) to a class of nonlinear systems.

Contrary to the problem of regional remediability, recently introduced for the same class of systems (Qaraai et al., 2008), which consists in neutralizing the effects of a spreadable disturbance on a given zone, the principle of the protector control of this zone consists in preventing disturbance effects to reach such a zone during the entire time interval of the evolution of the system considered (Qaraai et al., 2009).

Therefore, the study of the protector control will mainly take into account the trajectory of the property caused by the spreadable disturbance and also the first time at which such a property starts to reach the vulnerable zone. Indeed, if we do not act before this first time, then the area will be reached, and in this case we cannot consider its protection but rather its remediability. This means the need to control the trajectory of the disturbance effects so that it cannot pass through the fixed area.

To characterize solutions of this problem, the approach of a remediability of a certain nonlinear delay system will be introduced. The delay arising here is justified by the fact that we need to act "before" the disturbance starts to reach the vulnerable zone.

This work is structured as follows. After introducing the protector control problem, we review a few mathematical results used for a new formulation of this problem. The solutions to this problem are characterized using the approach of the remediability of a certain nonlinear delay system so that an algorithm will be given. Moreover, a second characterization will be given by coupling the families of transformations and delay. In the last section, we illustrate the concept of the control protector by considering a variant of Fisher's equation (Giuggioli and Kenkre, 2003) which can model the problem of bacterial dynamics.

\section{Problem statement: Definitions}

Let $\Omega$ be an open bounded subset of $\left.\mathbb{R}^{n}, I=\right] 0, T[\mathrm{a}$ time interval, $\sigma$ a fixed region in $\Omega$ and $\omega$ the geometrical support of a given disturbance $f$, which affects the initial condition of the system. Assume that meas $(\sigma) \neq 0$, $\bar{\omega} \cap \bar{\sigma}=\emptyset$ and the disturbance $f$ is spreadable from $\omega$ (Bernoussi , 2010). Consequently, if the zone $\sigma$ is $f$-vulnerable (Bernoussi and Amharref, 2003), it can be reached by the disturbance $f$.

In this work we consider the problem of the protector control, e.g., a control which can "protect" the vulnerable zone subjected to the disturbance during all the time interval $I$. The control considered will be determined through a measure function. This problem can be studied based on the compensation problem developed by Qaraai et al. (2008) for the same class of nonlinear systems.

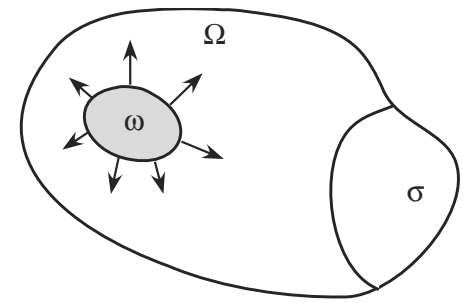

Fig. 1. Localization of $\omega$ and $\sigma$.

Consider a system governed by the following nonlinear state equation:

$$
(\widetilde{S})\left\{\begin{array}{l}
\dot{\tilde{z}}(t)=F \widetilde{z}(t)+B u(t), \quad 0<t<T, \\
\widetilde{z}(0)=z_{0}+f,
\end{array}\right.
$$

where $\widetilde{z} \in L^{p}(0, T ; Z), p>1, u \in L^{2}(0, T ; \mathcal{U}), f \in \mathcal{F}$, $B \in \mathcal{L}(\mathcal{U} ; Z)$ and $F: Z \longrightarrow Z$ is a nonlinear operator which satisfies the following assumptions:

1. $F(0)=0$

2. $F$ is continuously differentiable at every point $f$, where $f \in \mathcal{F} \subset Z$.

The state space $Z$, control $\mathcal{U}$ and disturbance $\mathcal{F}$ are separable Hilbert spaces.

Let $\chi_{\sigma}$ be the restriction to $\sigma$, and $\chi_{\sigma}^{*}$ the adjoint operator of $\chi_{\sigma}$ defined by

$$
\chi_{\sigma}^{*}: Z_{\sigma} \longrightarrow Z
$$

such that

$$
\left(\chi_{\sigma}^{*} z\right)(x)= \begin{cases}z(x) & \text { on } \sigma \\ 0 & \text { elsewhere }\end{cases}
$$

where $Z_{\sigma}$ is a subspace of $Z$, which designates the set of states restricted to $\sigma$ :

$$
Z_{\sigma}=\left\{\chi_{\sigma} z=z_{\left.\right|_{\sigma}}: z \in Z\right\} .
$$

The system (1) is augmented by the measure function given by

$$
(\widetilde{E}) \quad \widetilde{y}^{\sigma}(t)=C \chi_{\sigma}^{*} \chi_{\sigma} \widetilde{z}(t),
$$


with $\widetilde{y}^{\sigma} \in L^{p}(0, T ; Y), C \in \mathcal{L}(Z ; Y)$, and $Y$ is a selfadjoint Hilbert space $\left(Y=Y^{*}\right)$.

Remark 1. The operators $B$ and $C$ are assumed to be bounded. Otherwise, their images will be respectively $Z_{1}$ and $Y_{1}$ such that $Z \subset Z_{1} \subset Z^{*}$ and $Y \subset Y_{1} \subset Y^{*}$.

We assume that the system $(\widetilde{S})$ admits one unique solution $\widetilde{z}_{f, u}$, and we set

$$
\begin{cases}\widetilde{z}_{f, u} & \text { the solution of }(\widetilde{S}), \\ \widetilde{z}_{f, 0} & \text { the solution of }(\widetilde{S}) \text { when } f \neq 0 \text { and } u=0, \\ \widetilde{z}_{0,0} & \text { the solution of }(\widetilde{S}) \text { when } f=0 \text { and } u=0, \\ \widetilde{y}_{f, u}^{\sigma} & \text { the output }(\widetilde{E}), \\ \widetilde{y}_{f, 0}^{\sigma} & \text { the output }(\widetilde{E}) \text { when } f \neq 0 \text { and } u=0, \\ \widetilde{y}_{0,0}^{\sigma} & \text { the output }(\widetilde{E}) \text { when } f=0 \text { and } u=0 .\end{cases}
$$

Consider the property $\mathcal{P}$ and the subsets $w_{f, 0, t}$ of $\Omega$ defined respectively by

$$
\begin{aligned}
\mathcal{P} \widetilde{z}_{f, 0}(x, t) \Longleftrightarrow & \widetilde{z}_{f, 0}(x, t) \neq \widetilde{z}_{0,0}(x, t) \text { and } \\
& w_{f, 0, t}=\left\{x \in \Omega: \mathcal{P} \widetilde{z}_{f, 0}(x, t)\right\} .
\end{aligned}
$$

Here $w_{f, 0, t}$ is the set of all points $x$ in $\Omega$, where the state of the disturbed system $z_{f, 0}$ satisfies property $\mathcal{P}$ at time $t$ (e.g., the points where the state $z_{f, 0}$ is affected by the disturbance $f$ ). We recall the following definition (Bernoussi , 2010; Qaraai et al., 2008).

\section{Definition 1.}

1. We say that the disturbance $f$ is spreadable (respectively $\mathcal{A}$-spreadable $)$ if the family $\left(w_{f, 0, t}\right)_{t \in I}$ is increasing in the inclusion sense:

$$
w_{f, 0, t} \subset w_{f, 0, s}, \quad \forall t, s \in I: t \leq s,
$$

(resp. in the measure sense:

$$
\begin{aligned}
\mu\left(w_{f, 0, t} \backslash w_{f, 0, s}\right) \leq \mu\left(w_{f, 0, s} \backslash\right. & \left.w_{f, 0, t}\right), \\
& \forall t, s \in I: t \leq s .)
\end{aligned}
$$

2. The zone $\sigma$ is said to be $f$-vulnerable if there exists a time $t \in[0, T]$ such that

$$
w_{f, 0, t} \cap \stackrel{\circ}{\sigma} \neq \emptyset,
$$

where $\mu$ is the Lebesgue measure and $\stackrel{\circ}{\sigma}$ stands for the interior of $\sigma$.

3. If a zone $\sigma$ is $f$-vulnerable, then it is said to be $f$ remediable on $[0, T]$ if

$$
\widetilde{y}_{f, u}^{\sigma}(T)=\widetilde{y}_{0,0}^{\sigma}(T)
$$

In Definition 1 we consider the interior of $\sigma$ instead of $\sigma$ because its boundary $\Gamma_{\sigma}$ can be a natural barrier to the disturbance $f$.

For a given $z_{0} \in Z$, a disturbance $f \in \mathcal{F}$, and a measure function $(\widetilde{E})$, the protector control problem is formulated as follows:

$$
(\widetilde{P})\left\{\begin{array}{l}
\text { Find a control } u \in L^{2}(0, T ; \mathcal{U}) \text { such that } \\
\widetilde{y}_{f, u}^{\sigma}(t)=\widetilde{y}_{0,0}^{\sigma}(t), \quad \forall t \in[0, T] .
\end{array}\right.
$$

The protector control problem $(\widetilde{P})$ takes into account the zone $\sigma$, the normal evolution of system $\widetilde{y}_{0,0}^{\sigma}$ (an autonomous undisturbed case) and the evolution of the disturbed controlled system $\widetilde{y}_{f, u}^{\sigma}$ for every $t \in[0, T]$. This means that we must keep the disturbed system on its normal evolution during the whole time interval $[0, T]$ by a suitable control $u$. The role of such a control is to prevent effects of the disturbance $f$ to reach the zone $\sigma$ during the entire $[0, T]$. This is why the protection problem is different from the regional remediability one. Indeed, the principle of regional remediability (Afifi et al., 2002; Qaraai et al., 2008) consists in compensating, by an adequate control, the effects of the disturbance $f$ on the reached zone $\sigma$ at final time $T$. Notice that, in this case, the zone $\sigma$ is not only vulnerable, but it may also be reached by such disturbance effects. In other words, this consists in making the disturbed system on its normal evolution on $\sigma$ by a suitable control $u$ at time $T$. Recall that (Afifi et al., 2002; Qaraai et al., 2008) the regionally remediability problem on $\sigma$ is formulated as follows:

$$
(\widetilde{R P})\left\{\begin{array}{l}
\text { Find a control } u \in L^{2}(0, T ; \mathcal{U}) \text { such that } \\
\widetilde{y}_{f, u}^{\sigma}(T)=\widetilde{y}_{0,0}^{\sigma}(T) .
\end{array}\right.
$$

Assume that the system $(\widetilde{S})-(\widetilde{E})$ is $\sigma$-observable (Zerrik et al., 1994).

Definition 2. If a zone $\sigma$ is $f$-vulnerable, then

1. $\sigma$ is said to be $f$-protectable if

$$
\exists u \in L^{2}(0, T ; \mathcal{U}): \widetilde{y}_{f, u}^{\sigma}(t)=\widetilde{y}_{0,0}^{\sigma}(t), \quad \forall t \in[0, T]
$$

In this case $u$ is said to be an $f$ - $\sigma$-protector control.

2. $\sigma$ is said to be weakly $f$-protectable if

$$
\begin{aligned}
\forall \varepsilon>0, \exists u \in L^{2}(0, T ; \mathcal{U}): \\
\quad\left\|\widetilde{y}_{f, u}^{\sigma}(t)-\widetilde{y}_{0,0}^{\sigma}(t)\right\|_{Y} \leq \varepsilon, \quad \forall t \in[0, T] .
\end{aligned}
$$

We say that $u$ is a weakly $f$ - $\sigma$-protector control with a tolerance $\varepsilon$.

\section{Remark 2.}

1. As the system $(\widetilde{S})$ is nonlinear, the existence of the solution $\widetilde{z}_{f, u}$ (and, consequently, the observation $\widetilde{y}_{f, u}^{\sigma}$ ), depends particularly on the disturbance $f$. That is why we 
have considered the notion ' $f$-protectable' in Definition2, In the linear case we can consider the corresponding notion ' $\sigma$-protectable' in the sense that $\sigma$ is $f$-protectable for all $f \in \mathcal{F}$.

2. An $f$ - $\sigma$-protector control is an $f$ - $\sigma$-remediable one. The converse is not true.

3. If $\sigma$ is not $f$-vulnerable, then we do not have to protect nor remedy it. That is why we assume that the zone considered is vulnerable in Definition 2

4. Let $\omega$ be the geometrical support of the disturbance $f$. If $\bar{\omega} \cap \bar{\sigma} \neq \emptyset$ then $\sigma$ is reached by the effects of $f$, and in this case we cannot protect it, but it is possible to influence its remediability.

\section{Transformation of the problem $(\widetilde{\mathbf{P}})$}

The problem $(\widetilde{P})$ as it was formulated is not easy to approach. Indeed, in the expression for the solution $\widetilde{z}_{f, u}$ there is not any explicit relation of control $u$ and disturbance $f$, and, consequently, we cannot use directly the discussed approach used in the linear case (Bernoussi, 2010). To overcome these difficulties, we start by transforming the system (1) to an equivalent system $(S)$ around $f$, and we reformulate the problem $(\widetilde{P})$.

Let $\widetilde{z}$ be the solution of the system (1). With the state variable change

$$
z(t)=\widetilde{z}(t)-f
$$

the system becomes

$$
\left\{\begin{array}{l}
\dot{z}(t)=F(z(t)+f)+B u(t), \quad 0<t<T, \\
z(0)=z_{0}
\end{array}\right.
$$

Using Assumption 2 on $F$ we obtain, by linearizing around $f$,

$$
F(z(t)+f)=F(f)+F^{\prime}(f) z(t)+N_{f} z(t),
$$

which permits to express the system in a semilinear form

$$
(S)\left\{\begin{aligned}
\dot{z}(t) & =A_{f} z(t)+N_{f} z(t)+F f \\
& +B u(t), \quad 0<t<T, \\
z(0)= & z_{0},
\end{aligned}\right.
$$

where $A_{f}=F^{\prime}(f) \in \mathcal{L}(Z)$ and $N_{f}: Z \longrightarrow Z$ designates the nonlinearities term. On the other hand, the measure function $(\widetilde{E})$ around $f$ is given by

$$
\widetilde{y}^{\sigma}(t)=C \chi_{\sigma}^{*} \chi_{\sigma} \widetilde{z}(t)=C \chi_{\sigma}^{*} \chi_{\sigma}(z(t)+f) .
$$

Setting $y^{\sigma}(t)=\widetilde{y}^{\sigma}(t)-C \chi_{\sigma}^{*} \chi_{\sigma} f$ and using the linearity of $C \chi_{\sigma}^{*} \chi_{\sigma}$, the transformed measure function is expressed by

$$
(E) \quad y_{f, u}^{\sigma}(t)=C \chi_{\sigma}^{*} \chi_{\sigma} z_{f, u}(t),
$$

where $z_{f, u}$ is the solution of the system (12). Accordingly, in a neighborhood of $f$, we consider the following problem:

$$
(P)\left\{\begin{array}{l}
\text { Find a control } u \in L^{2}(0, T ; \mathcal{U}) \text { such that } \\
y_{f, u}^{\sigma}(t)=y_{0,0}^{\sigma}(t)-C \chi_{\sigma}^{*} \chi_{\sigma} f, \quad \forall t \in[0, T] .
\end{array}\right.
$$

It is clear that the problems $(\widetilde{P})$ and $(P)$ are equivalent in a neighborhood of $f$.

To treat the problem $(P)$, we rewrite it in an explicit form to display the control terms in $u$, the disturbance in $f$, and also those of nonlinearities in $z_{f, u}$.

3.1. Existence and uniqueness of the solution of $(S)$. We assume that the linear operator $A_{f}$ is closed, with a domain dense in $Z$, and generates a strongly continuous semigroup $\left(S_{f}(t)\right)_{t \geq 0}$. We prove that under some assumptions the system $(S)$ has a unique solution $z_{f, u}$ given by (Qaraai et al., 2008; Qaraai, 2008)

$$
\begin{aligned}
z_{f, u}(t)= & S_{f}(t) z_{0}+\int_{0}^{t} S_{f}(t-s) N_{f} z_{f, u}(s) \mathrm{d} s \\
& +\int_{0}^{t} S_{f}(t-s) F f \mathrm{~d} s \\
& +\int_{0}^{t} S_{f}(t-s) B u(s) \mathrm{d} s,
\end{aligned}
$$

which can be written in the following form:

$$
z_{f, u}(t)=S_{f}(t) z_{0}+\widehat{H}_{f, t} z_{f, u}+\widetilde{H}_{f, t} f+H_{f, t} u,
$$

where the three operators $\widehat{H}_{f, t}, \widetilde{H}_{f, t}$ and $H_{f, t}$ are defined respectively by

$$
\widehat{H}_{f, t}:\left\{\begin{aligned}
L^{p}(0, T ; Z) & \rightarrow Z \\
z & \mapsto \int_{0}^{t} S_{f}(t-s) N_{f} z(s) \mathrm{d} s
\end{aligned}\right.
$$

$$
\widetilde{H}_{f, t}:\left\{\begin{aligned}
\mathcal{F} & \rightarrow Z \\
f & \mapsto \int_{0}^{t} S_{f}(t-s) F f \mathrm{~d} s
\end{aligned}\right.
$$

$$
H_{f, t}:\left\{\begin{aligned}
L^{2}(0, T ; \mathcal{U}) & \rightarrow Z \\
u & \mapsto \int_{0}^{t} S_{f}(t-s) B u(s) \mathrm{d} s
\end{aligned}\right.
$$

Consider also the mapping $\psi(\cdot, f, u): z \in L^{p}(0, T ; Z) \longmapsto \psi(z, f, u) \in L^{p}(0, T ; Z)$ 
defined by

$\psi(z, f, u):\left\{\begin{array}{rlrl}{[0, T]} & \rightarrow & Z, \\ t & \mapsto z, f, u)(t)=S_{f}(t) z_{0} \\ & +\widehat{H}_{f, t} z+\widetilde{H}_{f, t} f+H_{f, t} u .\end{array}\right.$

Then, to prove that $z_{f, u}$ is given by (13), we prove that there exists $d>0$ such that the mapping $\psi(\cdot, f, u)$ admits one unique fixed point $z_{f, u}$ in the ball $B(0, d)$ of $L^{p}(0, T ; Z)$. Therefore, denote by $\|\cdot\|_{p}=\|\cdot\|_{L^{p}(0, T ; Z)}$, $p \in] 1,+\infty[$, and assume that (Qaraai et al., 2008)

(H1) $\exists g_{1} \in L^{q}(0, T)$ with $\frac{1}{q}+\frac{1}{p}=1$ :

$$
\left\|S_{f}(t)\right\|_{\mathcal{L}(Z)} \leq g_{1}(t), \quad \forall t \in[0, T]
$$

$(\mathrm{H} 2)\left\{\begin{array}{l}1 . N_{f}(0)=0, \\ 2 .\left\|N_{f} z_{1}(t)-N_{f} z_{2}(t)\right\|_{Z} \\ \quad \leq K\left(\left\|z_{1}\right\|_{p} ;\left\|z_{2}\right\|_{p}\right)\left\|z_{1}(t)-z_{2}(t)\right\|_{Z}, \\ \forall z_{1}, z_{2} \in L^{p}(0, T ; Z) ; K: \mathbb{R}_{+}^{2} \longrightarrow \mathbb{R}_{+}: \\ \lim _{\left(\theta_{1} ; \theta_{2}\right) \rightarrow(0,0)} K\left(\theta_{1} ; \theta_{2}\right)=0 .\end{array}\right.$

For some $a \in] 0,1[$ let

$$
\begin{aligned}
k & =\sup _{\theta<a} K(\theta ; 0), \\
K & =\sup _{\left\|\left(\theta_{1} ; \theta_{2}\right)\right\|_{\mathbb{R}_{+}^{2}<a}} K\left(\theta_{1} ; \theta_{2}\right),
\end{aligned}
$$$$
K^{*}=\max (k, K) \text {, }
$$

(H3) $\left\{\begin{aligned} \exists \alpha>0: & \left\|S_{f}(t) z_{0}\right\|_{Z} \leq \alpha\left\|z_{0}\right\|_{Z}, \\ & \forall t \in[0, T], \\ \exists \beta>0: & \left\|H_{f, t} u\right\|_{Z} \leq \beta\|u\|_{L^{2}(0, T ; \mathcal{U})}, \\ & \forall t \in[0, T], \\ \exists \gamma>0: & \left\|\widetilde{H}_{f, t} f\right\|_{Z} \leq \gamma\|f\|_{\mathcal{F}}, \forall t \in[0, T],\end{aligned}\right.$

(H4) $T<\frac{1}{\left(K^{*}\left\|g_{1}\right\|_{q}\right)^{p}}$,

(H5) $\exists \lambda \in] 0,1[$ :

$$
\left.\alpha\left\|z_{0}\right\|_{Z}+\gamma\|f\|_{\mathcal{F}} \in\right] 0, \frac{\lambda\left(1-T^{\frac{1}{p}} k\left\|g_{1}\right\|_{q}\right)}{T^{\frac{1}{p}}}[.
$$

Under all these assumptions, we have the following result (Qaraai et al., 2008).

Theorem 1. If the hypotheses (H1)-(H5) are satisfied, then

(a) There exists $d>0$ and $m=m(d)>0$ such that, for all $u$ in the ball $B(0, m)$ of $L^{2}(0, T ; \mathcal{U})$, the mapping $\psi(\cdot, f, u)$ admits one unique fixed point $z_{f, u}$ solution of $(S)$ in the ball $B(0, d)$ of $L^{p}(0, T ; Z)$.

(b) The mapping $h_{1}: u \in B(0, m) \longmapsto z_{f, u} \in B(0, d)$ is Lipschitzian.
Proof. (a) We show that there exists $d \in] 0,1[$ and $m=$ $m(d)>0$ with

$$
m=\frac{1}{\beta}\left[\frac{d\left(1-T^{\frac{1}{p}} k\left\|g_{1}\right\|_{q}\right)}{T^{\frac{1}{p}}}-\alpha\left\|z_{0}\right\|_{Z}-\gamma\|f\|_{\mathcal{F}}\right]_{(19)}
$$

such that for all $u \in B(0, m)$ we have $\psi(B(0, d), f, u) \subset$ $B(0, d)$. Besides, $\psi(\cdot, f, u)$ is a contraction in $B(0, d)$ with a contraction constant $\left.c_{1} \in\right] 0,1$,

$$
c_{1}=T^{\frac{1}{p}}\left\|g_{1}\right\|_{q} K .
$$

For the point (b), the Lipschitz constant $l$ is given by

$$
l=\frac{T^{\frac{1}{p}} \beta}{1-c_{1}}
$$

\section{Remark 3}

1. The hypothesis $(\mathrm{H} 1)$ is true for the whole strongly continuous semigroup $\left(S_{f}(t)\right)_{t \in I}$ such that $\left\|S_{f}(t)\right\|_{\mathcal{L}(Z)} \leq$ $M e^{w t}$ for some constants $M$ and $w$ (Curtain and Pritchard, 1978; Pazy, 1983).

2. For the hypothesis (H2), the nonlinear operator $N_{f}$ is assumed to be globally Lipschitzian with respect to $K^{*}$ in $Z$.

3. The positive constants $\alpha, \beta$ and $\gamma$ in the hypothesis (H3) are given respectively by

$$
\begin{gathered}
\alpha=\max _{t \in[0, T]} g_{1}(t), \\
\beta=\left\|g_{1}\right\|_{q}\|B\|, \\
\gamma=T^{\frac{1}{p}}\left\|g_{1}\right\|_{q}\|F\| .
\end{gathered}
$$

4. The hypotheses (H4) and (H5) are the conditions on the final time $T$, the initial condition $z_{0}$ and the disturbance $f$ for which the state $z_{f, u}$ given by (13) is defined for some control $u$. Besides, (H5) justifies the fact of considering in Remark 2 the difference between $\sigma$ being $f$-protectable and $\sigma$ being protectable for all $f$.

5. For all $t \in[0, T]$ let

$$
l(t)=N_{f} z_{f, u}(t)+F f+B u(t) .
$$

Then 13 becomes

$$
z_{f, u}(t)=S_{f}(t) z_{0}+\int_{0}^{t} S_{f}(t-s) l(s) \mathrm{d} s .
$$

It is shown (Curtain and Pritchard, 1978) that if $l \in$ $C^{1}([0, T], Z)$, then (13) is a classical solution of (12). Thus, to assure the regularity of the solution to (12), we assume moreover that $N_{f} z_{f, u}, B u \in C^{1}([0, T], Z)$. For more details, we refer the reader to the work of Henry (1981). 
3.2. New formulation of $(\widetilde{\boldsymbol{P}})$. Since the expression of the solution of $(S)$ in a neighborhood for $f$ is given by

$$
z_{f, u}(t)=S_{f}(t) z_{0}+\widehat{H}_{f, t} z_{f, u}+\widetilde{H}_{f, t} f+H_{f, t} u,
$$

the measure function $(E)$ will be

$(E) y_{f, u}^{\sigma}(t)=C \chi_{\sigma}^{*} \chi_{\sigma} S_{f}(t) z_{0}+C \chi_{\sigma}^{*} \chi_{\sigma} \widehat{H}_{f, t} z_{f, u}$ $+C \chi_{\sigma}^{*} \chi_{\sigma} \widetilde{H}_{f, t} f+C \chi_{\sigma}^{*} \chi_{\sigma} H_{f, t} u$.

In the case where $f=0$ and $u=0$, we have

$$
z_{0,0}(t)=S_{0}(t) z_{0}+\widehat{H}_{0, t} z_{0,0}
$$

Then the associated measure function is

$$
y_{0,0}^{\sigma}(t)=C \chi_{\sigma}^{*} \chi_{\sigma} S_{0}(t) z_{0}+C \chi_{\sigma}^{*} \chi_{\sigma} \widehat{H}_{0, t} z_{0,0} .
$$

Hence the problem $(P)$ is rewritten as follows:

$$
(P)\left\{\begin{array}{l}
\text { Find a control } u \in L^{2}(0, T ; \mathcal{U}) \text { such that } \\
C \chi_{\sigma}^{*} \chi_{\sigma} H_{f, t} u+C \chi_{\sigma}^{*} \chi_{\sigma} \widehat{H}_{f, t} z_{f, u} \\
+C \chi_{\sigma}^{*} \chi_{\sigma} \widetilde{H}_{f, t} f+C \chi_{\sigma}^{*} \chi_{\sigma} f \\
+C \chi_{\sigma}^{*} \chi_{\sigma}\left(S_{f}(t)-S_{0}(t)\right) z_{0} \\
-C \chi_{\sigma}^{*} \chi_{\sigma} \widehat{H}_{0, t} z_{0,0}=0, \quad \forall t \in[0, T] .
\end{array}\right.
$$

\section{Protector control approaches}

Note that, even if Problem $(P)$ involves control, disturbance and state terms $\left(u, f, z_{f, u}\right)$, its solution remains delicate due to the presence of an equality constraint for every $t \in[0, T]$. To overcome this difficulty and to give a link with a remediable control, we will introduce two approaches.

\subsection{Remediability and a delay system.}

4.1.1. Principle. In this section we will introduce a delay system whose state is defined according to the original system (12) to reduce the solving of Problem $(P)$ to that of the remediability problem of a resulting delay system. That will be done in the following three steps:

1. Introducing a suitable delay system from System $(S)$ : the protector control problem will be transformed to a remediable control one of the resulting delay system.

2. Formulation of the remediability problem for the resulting delay system.

3. Solution of the obtained problem.
Therefore, consider the Banach space $L^{p}(-T, 0, Z)$ on which, for all $t \in[0, T]$, the following strongly continuous semigroup $(R(t))_{t \geq 0}$ is defined:

$$
(R(t) b)(r)= \begin{cases}b(t+r), & r \in[-T,-t], \\ 0, & r \in]-t, 0] .\end{cases}
$$

The operator $D=\mathrm{d} / \mathrm{d} s$ is the infinitesimal generator of $(R(t))_{t>0}$ on $L^{p}(-T, 0 ; Z)$. Also define the mapping $G \in \mathcal{L}\left(Z, L^{p}(-T, 0 ; Z)\right)$ as follows:

$$
(G a(x))(r)=a(x), \quad \forall a(t) \in Z, \quad \forall r \in[-T, 0] .
$$

Then consider the operator $\Lambda$ defined by

$$
\Lambda:\left\{\begin{array}{lll}
Z & \longrightarrow & L^{p}(-T, 0 ; Z), \\
z_{f, u}(t) \longmapsto & \Lambda z_{f, u}(t)=\bar{z}_{f, u}(t) \\
& =R(t)\left(G z_{0}\right) \\
& -D \int_{0}^{t} R(t-s) G z_{f, u}(s) \mathrm{d} s,
\end{array}\right.
$$

where $z_{f, u}$ designates the solution of the system (12). Consequently, the associated measure function is given in $L^{p}(-T, 0 ; Y)$ by

$$
(\bar{E}): \quad \bar{y}_{f, u}^{\sigma}(t)=C \chi_{\sigma}^{*} \chi_{\sigma} \bar{z}_{f, u}(t), \quad t \in[0, T] .
$$

Recall the following result, which will be useful later.

Lemma 1. ( Ichikawa, 1982)

$$
\left(\bar{y}_{f, u}^{\sigma}(T)\right)(r)=y_{f, u}^{\sigma}(T+r), \quad \forall r \in[-T, 0] .
$$

Proof. The proof is based immediately on the expressions (25)-28).

On the other hand, the way that the control $u$ and disturbance $f$ appear in (27) leads us to the introduction of a new state equation on the product space $\bar{W}=Z \times$ $L^{p}(-T, 0, Z)$ in the following way:

$$
\begin{aligned}
\bar{w}_{f, u}(t)= & U_{f}(t) \bar{w}_{0}+\int_{0}^{t} U_{f}(t-s) \mathcal{N}_{f} \bar{w}_{f, u}(s) \mathrm{d} s \\
& +\int_{0}^{t} U_{f}(t-s) \mathcal{G} f \mathrm{~d} s \\
& +\int_{0}^{t} U_{f}(t-s) \mathcal{B} u(s) \mathrm{d} s,
\end{aligned}
$$

where

$$
\begin{aligned}
2 \bar{w}_{f, u} & =\left(z_{f, u}, \bar{z}_{f, u}\right)^{\operatorname{tr}}, & \bar{w}_{0} & =\left(z_{0}, G z_{0}\right)^{\operatorname{tr}}, \\
\mathcal{N}_{f} & =\left(N_{f}, 0\right)^{\operatorname{tr}}, & \mathcal{G} & =(F, 0)^{\operatorname{tr}}, \\
\mathcal{B} & =(B, 0)^{\operatorname{tr}} & &
\end{aligned}
$$


and $\left(U_{f}(t)\right)_{t \geq 0}$ is the strongly continuous semigroup on $\bar{W}$ defined by

$$
U_{f}(t)=\left(\begin{array}{cc}
U_{0, f}(t) & 0 \\
U_{1, f}(t) & U_{2, f}(t)
\end{array}\right),
$$

where

$$
\begin{aligned}
& U_{0, f}(t)=S_{f}(t), \quad U_{2, f}(t)=R(t), \\
& U_{1, f}(t)=-D \int_{0}^{t} R(t-s) G S_{f}(s) \mathrm{d} s .
\end{aligned}
$$

If we designate by $\bar{A}_{f}$ the infinitesimal generator of the semigroup $\left(U_{f}(t)\right)_{t \geq 0}$ on the space $\bar{W}$, then the system associated with (30) is given by

$$
\left\{\begin{array}{l}
\dot{\bar{w}}_{f, u}(t)=\bar{A}_{f} \bar{w}_{f, u}(t)+\mathcal{N}_{f} \bar{w}_{f, u}(t)+\mathcal{G} f+\mathcal{B} u(t), \\
\quad 0<t<T, \\
\bar{w}(0)=\bar{w}_{0}
\end{array}\right.
$$

which is augmented by the measure function

$$
(\overline{\bar{E}}) \quad \overline{\bar{y}}_{f, u}^{\sigma}(t)=\overline{\bar{C}} \chi_{\sigma}^{*} \chi_{\sigma} \bar{w}_{f, u}(t),
$$

where

$$
\overline{\bar{C}}=\left(\begin{array}{cc}
C & 0 \\
0 & C
\end{array}\right)
$$

Remark that

$$
\overline{\bar{y}}_{f, u}^{\sigma}(t)=\left(y_{f, u}^{\sigma}(t), \bar{y}_{f, u}^{\sigma}(t)\right)^{\operatorname{tr}} \in Y \times L^{p}(-T, 0 ; Y) .
$$

Concerning (33), it is a state equation with an related delay system. We define (formally), through 27), the following operator:

$$
\Pi: \bar{z}_{f, u}(t) \in L^{p}(-T, 0 ; Z) \longmapsto z_{f, u}(t) \in Z .
$$

This permits us to introduce some new operators on the state $\bar{z}_{f, u}(T)$, the disturbance $f$ and the control $u$ which are defined by (where $L=L^{p}(-T, 0 ; Z)$ )

$$
\begin{aligned}
& \widehat{\mathcal{H}}_{f, T}:\left\{\begin{aligned}
L & \rightarrow L, \\
\bar{z}_{f, u}(T) & \mapsto p_{2} \int_{0}^{T} U_{f}(T-s) \mathcal{N}_{f} \Pi \bar{z}_{f, u}(s) \mathrm{d} s,
\end{aligned}\right. \\
& \widetilde{\mathcal{H}}_{f, T}:\left\{\begin{array}{l}
\mathcal{F} \rightarrow L, \\
f \mapsto p_{2} \int_{0}^{T} U_{f}(T-s) \mathcal{G} f \mathrm{~d} s,
\end{array}\right. \\
& \mathcal{H}_{f, T}:\left\{\begin{aligned}
L^{2}(0, T ; \mathcal{U}) & \rightarrow L, \\
u & \mapsto p_{2} \int_{0}^{T} U_{f}(T-s) \mathcal{B} u(s) \mathrm{d} s
\end{aligned}\right.
\end{aligned}
$$

where

$$
p_{2}:\left\{\begin{aligned}
\bar{W} & \rightarrow L \\
\left(z_{f, u}(t), \bar{z}_{f, u}(t)\right) & \mapsto \bar{z}_{f, u}(t) .
\end{aligned}\right.
$$

Using (30), 31) and 37, we find that

$$
\begin{aligned}
\widehat{\mathcal{H}}_{f, T} \bar{z}_{f, u}(T) & =\int_{0}^{T} U_{1, f}(T-s) N_{f} \Pi \bar{z}_{f, u}(s) \mathrm{d} s \\
\widetilde{\mathcal{H}}_{f, T} f & =\int_{0}^{T} U_{1, f}(T-s) F f \mathrm{~d} s \\
\mathcal{H}_{f, T} u & =\int_{0}^{T} U_{1, f}(T-s) B u(s) \mathrm{d} s
\end{aligned}
$$

where $\left(U_{1, f}(t)\right)_{t \geq 0}$ is given by (32).

Remark 4. The determination of the operator $\Pi$ given in (36) is not necessary. It is introduced just to have an explicit dependence on (38). We clarify this point later.

We define below three operators depending respectively on $\bar{z}_{f, u}(T), f, u$ and the final time $T$. This permits us to consider the following problem:

$(\bar{P})\left\{\begin{array}{l}\text { Find } u \in L^{2}(0, T ; \mathcal{U}) \text { such that } \\ C \chi_{\sigma}^{*} \chi_{\sigma} G\left[S_{f}(T)-S_{0}(T)\right] z_{0}-C \chi_{\sigma}^{*} \chi_{\sigma} \widehat{\mathcal{H}}_{0, T} \bar{z}_{0,0} \\ +C \chi_{\sigma}^{*} \chi_{\sigma} \widetilde{\mathcal{H}}_{f, T} f+C \chi_{\sigma}^{*} \chi_{\sigma} D \int_{0}^{T} R(T-s) G f \mathrm{~d} s \\ +C \chi_{\sigma}^{*} \chi_{\sigma} \mathcal{H}_{f, T} u+C \chi_{\sigma}^{*} \chi_{\sigma} \widehat{\mathcal{H}}_{f, T} \bar{z}_{f, u}(T)=0 .\end{array}\right.$

From this formulation and Definition 2 of an $f-\sigma$ protector control of a given $f$-vulnerable zone $\sigma$, we have the following characterization.

Theorem 2. If $\sigma$ is $f$-vulnerable, then $u$ is an $f$ - $\sigma$ protector control if and only if $u$ is a solution to $(\bar{P})$.

Remark 5. The solution to $(\bar{P})$ is a remediable control for the resulting delay system. This means that the protection of $\sigma$ can be seen as a prediction/correction problem when we assume that this area can be reached in the future (at the final time $T$ ), then to the remediability of $\sigma$ at final time $T$ we apply a delay in time $r \in[-T, 0]$ to return to the current situation $t=T+r \in[0, T]$ (e.g., real time or system without delay).

Proof. (Theorem 2) We prove that Problems $(\bar{P})$ and $(P)$ are equivalent. Indeed, let $u \in L^{2}(0, T ; \mathcal{U})$ and $f \in \mathcal{F}$. Through (27), we have

$$
\bar{z}_{f, u}(T)=R(T)\left(G z_{0}\right)-D \int_{0}^{T} R(T-s) G z_{f, u}(s) \mathrm{d} s .
$$


Replacing $z_{f, u}$ by its expression (13), we obtain

$$
\begin{aligned}
\bar{z}_{f, u}(T) \\
=R(T)\left(G z_{0}\right)-D \int_{0}^{T} R(T-s) G\left[S_{f}(s) z_{0}\right. \\
\quad+\int_{0}^{s} S_{f}(s-q) N_{f} z_{f, u}(q) \mathrm{d} q \\
\quad+\int_{0}^{s} S_{f}(s-q) F f \mathrm{~d} q \\
\left.\quad+\int_{0}^{s} S_{f}(s-q) B u(s) \mathrm{d} q\right] \mathrm{d} s .
\end{aligned}
$$

From the expressions of the semigroup $\left(U_{1, f}(t)\right)_{t \geq 0}$, the operator $\Pi$ and the three operators given respectively by (32), (36), (38), 39) and (40), we obtain

$\bar{z}_{f, u}(T)$

$$
=G S_{f}(T) z_{0}+\widehat{\mathcal{H}}_{f, T} \bar{z}_{f, u}(T)+\widetilde{\mathcal{H}}_{f, T} f+\mathcal{H}_{f, T} u .
$$
comes

Hence the output function $(\bar{E})$ given by $(28)$ be-

$$
\begin{aligned}
\bar{y}_{f, u}^{\sigma}(T)=C \chi_{\sigma}^{*} \chi_{\sigma}\left(G S_{f}(T) z_{0}\right. & +\widehat{\mathcal{H}}_{f, T} \bar{z}_{f, u}(T) \\
& \left.+\widetilde{\mathcal{H}}_{f, T} f+\mathcal{H}_{f, T} u\right) .
\end{aligned}
$$

In the case where $f=0$ and $u=0$, we have

$$
\bar{y}_{0,0}^{\sigma}(T)=C \chi_{\sigma}^{*} \chi_{\sigma}\left(G S_{0}(T) z_{0}+\widehat{\mathcal{H}}_{0, T} \bar{z}_{0,0}(T)\right) .
$$

Then the control $u$ is a solution to $(\bar{P})$ if and only if $u$ satisfies

$$
\begin{aligned}
& C \chi_{\sigma}^{*} \chi_{\sigma} G\left[S_{f}(T)-S_{0}(T)\right] z_{0}-C \chi_{\sigma}^{*} \chi_{\sigma} \widehat{\mathcal{H}}_{0, T} \bar{z}_{0,0} \\
& +C \chi_{\sigma}^{*} \chi_{\sigma} \widetilde{\mathcal{H}}_{f, T} f+C \chi_{\sigma}^{*} \chi_{\sigma} D \int_{0}^{T} R(T-s) G f \mathrm{~d} s \\
& +C \chi_{\sigma}^{*} \chi_{\sigma} \mathcal{H}_{f, T} u+C \chi_{\sigma}^{*} \chi_{\sigma} \widehat{\mathcal{H}}_{f, T} \bar{z}_{f, u}(T)=0,
\end{aligned}
$$

which is equivalent to

$$
\bar{y}_{f, u}^{\sigma}(T)=\bar{y}_{0,0}^{\sigma}(T)-C \chi_{\sigma}^{*} \chi_{\sigma} D \int_{0}^{T} R(T-s) G f \mathrm{~d} s,
$$

which is also equivalent to

$$
\begin{aligned}
\left(\bar{y}_{f, u}^{\sigma}(T)\right)(r) & =\left(\bar{y}_{0,0}^{\sigma}(T)\right)(r) \\
& -\left(C \chi_{\sigma}^{*} \chi_{\sigma} D \int_{0}^{T} R(T-s) G f \mathrm{~d} s\right)(r),
\end{aligned}
$$

$\forall r \in[-T, 0]$. On the one hand, due to 25) and (26) we have

$$
\left(C \chi_{\sigma}^{*} \chi_{\sigma} D \int_{0}^{T} R(T-s) G f \mathrm{~d} s\right)(r)=C \chi_{\sigma}^{*} \chi_{\sigma} f .
$$

Using Lemma1 we obtain

$$
\left(\bar{y}_{f, u}^{\sigma}(T)\right)(r)=y_{0,0}^{\sigma}(T+r), \quad \forall r \in[-T, 0] .
$$

Then $u$ is a solution to $(\bar{P})$ if and only if $y_{f, u}^{\sigma}(T+r)=y_{0,0}^{\sigma}(T+r)-C \chi_{\sigma}^{*} \chi_{\sigma} f, \quad \forall r \in[-T, 0]$.

Setting $t=T+r \in[0, T]$, we obtain

$$
y_{f, u}^{\sigma}(t)=y_{0,0}^{\sigma}(t)-C \chi_{\sigma}^{*} \chi_{\sigma} f, \quad \forall t \in[0, T] .
$$

This is equivalent to the fact that $u$ is a solution to Problem $(P)$.

The point of Theorem 2 is to reduce the solving of the protector control problem $(P)$ defined for all times $t \in I$ to the one of the remediability problem $(\bar{P})$ where we consider only the final time $T$ and not the state constraint for every $t \in[0, T]$. In fact, we will be interested in solving Problem $(\bar{P})$ based on the results developed by Qaraai et al. (2008), but in this case the corresponding operators and functional spaces are related to a delay system.

4.1.2. Determination of the protector control. Using pseudoinverse techniques, the problem of the determination of a suitable control can be brought back to the problem of the determination of a fixed point of a certain mapping. Thus, and under some hypothesis, a sequence of controls will be generated by an algorithm convergent to the desired solution.

Indeed, the control $u$ is a solution to $(\bar{P})$ if it satisfies

$$
\begin{aligned}
& C \chi_{\sigma}^{*} \chi_{\sigma} G\left[S_{f}(T)-S_{0}(T)\right] z_{0}-C \chi_{\sigma}^{*} \chi_{\sigma} \widehat{\mathcal{H}}_{0, T} \bar{z}_{0,0} \\
& +C \chi_{\sigma}^{*} \chi_{\sigma} \widetilde{\mathcal{H}}_{f, T} f+C \chi_{\sigma}^{*} \chi_{\sigma} D \int_{0}^{T} R(T-s) G f \mathrm{~d} s \\
& \quad+C \chi_{\sigma}^{*} \chi_{\sigma} \mathcal{H}_{f, T} u+C \chi_{\sigma}^{*} \chi_{\sigma} \widehat{\mathcal{H}}_{f, T} \bar{z}_{f, u}(T)=0 .
\end{aligned}
$$

Consider the subset $V=\operatorname{Im}\left(C \chi_{\sigma}^{*} \chi_{\sigma} \mathcal{H}_{f, T}\right)$ of $L^{p}(-T, 0, Y)$ and set

$$
\bar{y}_{f}^{\sigma}(T)=C \chi_{\sigma}^{*} \chi_{\sigma} G\left[S_{f}(T)-S_{0}(T)\right] z_{0}
$$

$$
\begin{aligned}
& -C \chi_{\sigma}^{*} \chi_{\sigma} \widehat{\mathcal{H}}_{0, T} \bar{z}_{0,0}+C \chi_{\sigma}^{*} \chi_{\sigma} \widetilde{\mathcal{H}}_{f, T} f \\
& +C \chi_{\sigma}^{*} \chi_{\sigma} D \int_{0}^{T} R(T-s) G f \mathrm{~d} s .
\end{aligned}
$$

Then we seek a control $u$ such that

$$
C \chi_{\sigma}^{*} \chi_{\sigma} \mathcal{H}_{f, T} u+C \chi_{\sigma}^{*} \chi_{\sigma} \widehat{\mathcal{H}}_{f, T} \bar{z}_{f, u}(T)=\bar{y}_{f}^{\sigma}(T) .
$$

Using (38), we have

$$
\begin{aligned}
& C \chi_{\sigma}^{*} \chi_{\sigma} \mathcal{H}_{f, T} u \\
& =\bar{y}_{f}^{\sigma}(T)-\int_{0}^{T} C \chi_{\sigma}^{*} \chi_{\sigma} U_{1, f}(T-s) N \Pi \bar{z}_{f, u}(s) \mathrm{d} s .
\end{aligned}
$$


As $\Pi \bar{z}_{f, u}(t)=h_{1} u(t)$ (Eqn. (36) and part (b) of Theorem 3.1$)$, we have to find a control $u \in B(0, m)$ such that

$$
\begin{aligned}
& C \chi_{\sigma}^{*} \chi_{\sigma} \mathcal{H}_{f, T} u \\
& =\bar{y}_{f}^{\sigma}(T)-\int_{0}^{T} C \chi_{\sigma}^{*} \chi_{\sigma} U_{1, f}(T-s) N_{f} h_{1} u(s) \mathrm{d} s .
\end{aligned}
$$

where the nonlinear term is zero $\left(N_{f}=0\right)$ and, for $\bar{y}_{f}^{\sigma}(T) \in V$, the set

$$
\left\{u: C \chi_{\sigma}^{*} \chi_{\sigma} \mathcal{H}_{f, T} u=\bar{y}_{f}^{\sigma}(T)\right\}
$$

is a nonempty, closed and convex subset of $L^{2}(0, T ; \mathcal{U})$ which is a reflexive Banach space. Then there exists a unique control $u^{*}$ with a minimum norm, which satisfies

$$
C \chi_{\sigma}^{*} \chi_{\sigma} \mathcal{H}_{f, T} u^{*}=\bar{y}_{f}^{\sigma}(T) .
$$

Let $u^{*}=L \bar{y}_{f}^{\sigma}(T)$. Then the linear operator $L$ which applies $V$ in $L^{2}(0, T ; \mathcal{U})$ is the pseudo inverse of $C \chi_{\sigma}^{*} \chi_{\sigma} \mathcal{H}_{f, T}$. It is given by

$$
L=\mathcal{H}_{f, T}^{*} \chi_{\sigma}^{*} \chi_{\sigma} C^{*}\left[C \chi_{\sigma}^{*} \chi_{\sigma} \mathcal{H}_{f, T} \mathcal{H}_{f, T}^{*} \chi_{\sigma}^{*} \chi_{\sigma} C^{*}\right]^{-1} .
$$

Then on the space $V$ we can define the norm

$$
\|(\cdot)\|_{V}=\|L(\cdot)\|_{L^{2}(0, T ; \mathcal{U})} .
$$

It is on the Banach space $\left(V,\|\cdot\|_{V}\right)$ that we will consider Problem $(\bar{P})$.

On the other hand, we have

$$
\int_{0}^{T} C \chi_{\sigma}^{*} \chi_{\sigma} U_{1, f}(T-s) N_{f} h_{1} u(s) \mathrm{d} s \in V,
$$

because $C \chi_{\sigma}^{*} \chi_{\sigma} U_{1, f}(t) \in \mathcal{L}(Z, V)$. Then we must find a control $u \in B(0, m)$ such that

$$
\begin{aligned}
u=L & \left(\bar{y}_{f}^{\sigma}(T)\right. \\
& \left.-\int_{0}^{T} C \chi_{\sigma}^{*} \chi_{\sigma} U_{1, f}(T-s) N_{f} h_{1} u(s) \mathrm{d} s\right) .
\end{aligned}
$$

For $y_{f}^{\sigma} \in V$, consider the mapping

$$
\varphi\left(y_{f}^{\sigma}, \cdot\right): L^{2}(0, T ; \mathcal{U}) \rightarrow L^{2}(0, T ; \mathcal{U})
$$

defined by

$$
\begin{aligned}
\varphi\left(\bar{y}_{f}^{\sigma}(T), u\right) \\
=L\left(\bar{y}_{f}^{\sigma}(T)\right. \\
\left.\quad-\int_{0}^{T} C \chi_{\sigma}^{*} \chi_{\sigma} U_{1, f}(T-s) N_{f} h_{1} u(s) \mathrm{d} s\right) .
\end{aligned}
$$

Then, to prove that Problem $(\bar{P})$ has a solution, is equivalent, under some hypothesis, to proving that the mapping $\varphi\left(y_{f}^{\sigma}, \cdot\right)$ admits a unique fixed point $u^{*}$ in the ball $B(0, m)$ of $L^{2}(0, T ; \mathcal{U})$. For that and in addition to the hypotheses $(H 1)-(H 5)$, we assume that
(H6) $\exists g_{2} \in L^{q}(0, T)$ :

$$
\left\|C \chi_{\sigma}^{*} \chi_{\sigma} U_{1, f}(t)\right\|_{\mathcal{L}(Z, V)} \leq g_{2}(t), \quad \forall t \in[0, T] .
$$

(H7) $T<\frac{1}{\left(K^{*}\left\|g_{1}\right\|_{q}+\beta K^{*}\left\|g_{2}\right\|_{q}\right)^{p}}$.

(H8) $\left.\exists \lambda^{\prime} \in\right] 0,1\left[: \alpha\left\|z_{0}\right\|_{X}+\gamma\|f\|_{\mathcal{F}} \in\right.$

$$
] 0, \frac{\lambda^{\prime}\left(1-T^{\frac{1}{p}}\left(k\left\|g_{1}\right\|_{q}+\beta k\left\|g_{2}\right\|_{q}\right)\right)}{T^{\frac{1}{p}}}[\text {. }
$$

Then, using a Banach fixed point theorem (Smart, 1974), we have the following result:

Theorem 3. If the hypotheses $(H 1)-(H 8)$ are satisfied, then there exist $d$ and $\rho=\rho(d)>0$ such that for all $\bar{y}_{f}^{\sigma}(T) \in B(0, \rho)$, where $\bar{y}_{f}^{\sigma}(T)$ is given by (41), the mapping $\varphi\left(\bar{y}_{f}^{\sigma}(T), \cdot\right)$ admits a unique fixed point $u^{*}$ in $B(0, m)$.

Proof. The second condition of the hypothesis $(\mathrm{H} 2)$, $\lim _{\left(\theta_{1} ; \theta_{2}\right) \rightarrow(0,0)} K\left(\theta_{1} ; \theta_{2}\right)=0$, implies that

$$
\forall \varepsilon>0, \quad \exists d \in] 0,1\left[\sup _{\left\|\left(\theta_{1} ; \theta_{2}\right)\right\|<d} K\left(\theta_{1} ; \theta_{2}\right)<\varepsilon .\right.
$$

For

$$
\varepsilon=\frac{1}{T^{\frac{1}{p}}\left(\left\|g_{1}\right\|_{q}+\beta\left\|g_{2}\right\|_{q}\right)}
$$

and using the hypothesis $(H 7)$, we have

$$
\left.c_{2}=T^{\frac{1}{p}}\left(\left\|g_{1}\right\|_{q}+\beta\left\|g_{2}\right\|_{q}\right) K \in\right] 0,1[.
$$

Now we prove that $\varphi\left(\bar{y}_{f}^{\sigma}(T), B(0, m)\right) \subset B(0, m)$ for $\bar{y}_{f}^{\sigma}(T) \in V$, with $m$ and $\bar{y}_{f}^{\sigma}(T)$ given respectively by (19) and (41). Indeed, for $u \in B(0, m)$, we have

$$
\begin{aligned}
& \left\|\varphi\left(\bar{y}_{f}^{\sigma}(T), u\right)\right\|_{L^{2}(0, T ; \mathcal{U})} \\
& =\left\|\bar{y}_{f}^{\sigma}(T)-\int_{0}^{T} C \chi_{\sigma}^{*} \chi_{\sigma} U_{1, f}(T-s) N_{f} h_{1} u(s) \mathrm{d} s\right\|_{V} \\
& \leq\left\|\bar{y}_{f}^{\sigma}(T)\right\|_{V} \\
& \quad+\left\|\int_{0}^{T} C \chi_{\sigma}^{*} \chi_{\sigma} U_{1, f}(T-s) N_{f} h_{1} u(s) \mathrm{d} s\right\|_{V} .
\end{aligned}
$$

Using the hypotheses $(H 2)$ and $(H 6)$, we obtain $\| \varphi\left(\bar{y}_{f}^{\sigma}(T) \|_{L^{2}(0, T ; \mathcal{U})}\right.$

$$
\leq\left\|\bar{y}_{f}^{\sigma}(T)\right\|_{V}+\left\|g_{2}\right\|_{q} K\left(\left\|h_{1}(u)\right\|_{p} ; 0\right)\left\|h_{1}(u)\right\|_{p} .
$$

The fact that $u \in B(0, m)$ implies that $h_{1}(u) \in$ $B(0, d)$, and, using the constant $k$ given by 19 , we obtain

$$
\left\|\varphi\left(\bar{y}_{f}^{\sigma}(T), u\right)\right\|_{L^{2}(0, T ; \mathcal{U})} \leq\left\|\bar{y}_{f}^{\sigma}(T)\right\|_{V}+k\left\|g_{2}\right\|_{q} d .
$$


Then the condition

$$
u \in B(0, m) \Rightarrow \varphi\left(\bar{y}_{f}^{\sigma}(T), u\right) \in B(0, m)
$$

is satisfied if

$$
\left\|\bar{y}_{f}^{\sigma}(T)\right\|_{V} \leq m-d k\left\|g_{2}\right\|_{L^{1}(0, T)} .
$$

Using (19), we obtain $\left\|\bar{y}_{f}^{\sigma}(T)\right\|_{V} \leq \rho$ with

$$
\begin{aligned}
\rho= & \frac{d\left(1-T^{\frac{1}{p}}\left(k\left\|g_{1}\right\|_{q}+\beta k\left\|g_{2}\right\|_{q}\right)\right)}{\beta T^{\frac{1}{p}}} \\
& -\frac{\alpha\left\|z_{0}\right\|_{X}-\gamma\|f\|_{\mathcal{F}}}{\beta} .
\end{aligned}
$$

As a consequence of the hypothesis $(H 7)$, we have $1-T^{\frac{1}{p}}\left(k\left\|g_{1}\right\|_{q}+\beta k\left\|g_{2}\right\|_{q}\right)>0$. In addition to the hypothesis (H8) with $\lambda^{\prime}=d$, we obtain $\rho>0$. Thus, if $y_{f}^{\sigma} \in B(0, \rho)$, we get $\varphi\left(y_{f}^{\sigma}, B(0, m)\right) \subset B(0, m)$.

On the other hand, for all $u, v \in B(0, m)$ and $\bar{y}_{f}^{\sigma}(T) \in V$, we have

$$
\begin{aligned}
\varphi\left(\bar{y}_{f}^{\sigma}(T), u\right)-\varphi\left(\bar{y}_{f}^{\sigma}(T), v\right) \\
=L\left(\int_{0}^{T} C \chi_{\sigma}^{*} \chi_{\sigma} U_{1, f}(T-s) N_{f} h_{1} u(s) \mathrm{d} s\right) \\
\quad-L\left(\int_{0}^{T} C \chi_{\sigma}^{*} \chi_{\sigma} U_{1, f}(T-s) N_{f} h_{1} v(s) \mathrm{d} s\right) .
\end{aligned}
$$

Using the norm on $L^{2}(0, T ; \mathcal{U})$ and with the formula (44), we have

$$
\begin{gathered}
\left\|\varphi\left(\bar{y}_{f}^{\sigma}(T), u\right)-\varphi\left(\bar{y}_{f}^{\sigma}(T), v\right)\right\|_{L^{2}(0, T ; \mathcal{U})} \\
=\| \int_{0}^{T} C \chi_{\sigma}^{*} \chi_{\sigma} U_{1, f}(T-s) \\
{\left[N_{f} h_{1} u(s) d s-N_{f} h_{1} v(s)\right] \mathrm{d} s \|_{V} .}
\end{gathered}
$$

Then, by the hypotheses $(H 2)$ and $(H 6)$, we obtain

$$
\begin{aligned}
\left\|\varphi\left(\bar{y}_{f}^{\sigma}(T), u\right)-\varphi\left(\bar{y}_{f}^{\sigma}(T), v\right)\right\|_{L^{2}(0, T ; \mathcal{U})} & \\
& \leq\left\|g_{2}\right\|_{q} K\left\|h_{1}(u)-h_{1}(v)\right\|_{p} .
\end{aligned}
$$

As the mapping $h_{1}$ is Lipschitzian with respect to

$$
l=\frac{T^{\frac{1}{p}} \beta}{1-c_{1}},
$$

we have

$$
\begin{aligned}
\| \varphi\left(\bar{y}_{f}^{\sigma}(T), u\right)-\varphi\left(\bar{y}_{f}^{\sigma}(T), v\right) & \|_{L^{2}(0, T ; \mathcal{U})} \\
& \leq c_{3}\|u-v\|_{L^{2}(0, T ; \mathcal{U})}
\end{aligned}
$$

with

$$
c_{3}=\frac{T^{\frac{1}{p}} \beta\left\|g_{2}\right\|_{q} K}{1-c_{1}} .
$$

But $0 \leq c_{3}<1$ because

$$
\left.1-c_{3}=\frac{1-c_{2}}{1-c_{1}} \in\right] 0,1[.
$$

Then the mapping $\varphi\left(y_{f}^{\sigma}, \cdot\right)$ is a contraction one on $B(0, m)$. Thus for all $\bar{y}_{f}^{\sigma}(T) \in B(0, \rho)$, the mapping $\varphi\left(\bar{y}_{f}^{\sigma}(T), \cdot\right)$ has a unique fixed point $u^{*}$ in $B(0, m)$.

4.1.3. Implementation of the protector control. Based on the previous sections, we will give an algorithm which permits the generation of a control sequence that converges to the solution $u^{*}$ to Problem $(\bar{P})$. Based on Theorem 3, we have the following result:

Proposition 1. If the assumptions of Theorem 3 are satisfied and if $\bar{y}_{f}^{\sigma}(T) \in V$ such that $\left\|\bar{y}_{f}^{\sigma}(T)\right\|_{V} \leq \rho$, then the sequence defined by

$$
\left\{\begin{array}{l}
u_{0}=0 \\
u_{n+1}=\varphi\left(\bar{y}_{f}^{\sigma}(T), u_{n}\right)=L \bar{y}_{f}^{\sigma}(T) \\
-L \int_{0}^{T} C \chi_{\sigma}^{*} \chi_{\sigma} U_{1, f}(T-s) N_{f} h_{1} u_{n}(s) \mathrm{d} s
\end{array}\right.
$$

converges to the solution $u^{*}$ to $(\bar{P})$ in $L^{2}(0, T, \mathcal{U})$ and satisfies

$$
\left\|u_{n+1}-u_{n}\right\|_{L^{2}(0, T ; \mathcal{U})} \leq c_{3}^{n}\left\|u_{1}\right\| .
$$

Proof. The result follows from Theorem 3 and the properties of the fixed point theorem.

For all $n \in \mathbb{N}$, setting

$$
\bar{y}_{f, u_{n}}^{\sigma, n}(T)=C \chi_{\sigma}^{*} \chi_{\sigma} \bar{z}_{f, u_{n}}^{n}(T)
$$

and

$$
\bar{r}_{n+1}=\bar{y}_{f}^{\sigma}(T)-C \chi_{\sigma}^{*} \chi_{\sigma} \widehat{\mathcal{H}}_{f, T} \bar{z}_{f, u_{n}}^{n}(T),
$$

we obtain

$$
\begin{aligned}
\bar{y}_{f, u_{n}}^{\sigma, n}(T)= & \bar{y}_{f}^{\sigma}(T)-\bar{y}_{f}^{\sigma}(T)+C \chi_{\sigma}^{*} \chi_{\sigma}\left[G S_{f}(T) z_{0}\right. \\
& \left.+\widehat{\mathcal{H}}_{f, T} \bar{z}_{f, u_{n}}(T)+\widetilde{\mathcal{H}}_{f, T} f+\mathcal{H}_{f, T} u_{n}\right] .
\end{aligned}
$$

Using the expression for $\bar{y}_{f}^{\sigma}(T)$ given by (41), we have

$$
\bar{y}_{f, u_{n}}^{\sigma, n}(T)
$$

$$
\begin{aligned}
= & \bar{y}_{0,0}^{\sigma}(T)-C \chi_{\sigma}^{*} \chi_{\sigma} D \int_{0}^{T} R(T-s) G f \mathrm{~d} s \\
& +\bar{r}_{n}-\bar{r}_{n+1} .
\end{aligned}
$$


Thus

$$
\begin{aligned}
\bar{r}_{n+1}= & \bar{r}_{n}+\bar{y}_{0,0}^{\sigma}(T)-\bar{y}_{f, u_{n}}^{\sigma, n}(T) \\
& -C \chi_{\sigma}^{*} \chi_{\sigma} D \int_{0}^{T} R(T-s) G f \mathrm{~d} s .
\end{aligned}
$$

Moreover, it is easy to see that

$$
\begin{aligned}
& \| \bar{y}_{0,0}^{\sigma, n}(T)-\bar{y}_{f, u_{n}}^{\sigma}(T) \\
& \quad-C \chi_{\sigma}^{*} \chi_{\sigma} D \int_{0}^{T} R(T-s) G f \mathrm{~d} s\left\|_{V} \leq c_{3}^{n}\right\| u_{1} \| .
\end{aligned}
$$

Consequently we have the following algorithm.

\section{Algorithm 1.}

1. Data: $\Omega, \sigma, T, A, N, z_{0}, f, B, C, \varepsilon$.

2. Autonomous undisturbed case: determine $\bar{y}_{0,0}^{\sigma}(T)$.

3. Disturbed uncontrolled case: determine $\bar{y}_{f, 0}^{\sigma}(T)$.

4. Set $\bar{r}_{0}=0$ and consider $\bar{r}_{1}$ : formula (51) with $n=0$.

5. Determine: $u_{n}=L \bar{r}_{n}(L$ is given by (43) $)$.

6. Disturbed controlled case: determine $\bar{y}_{f, u_{n}}^{\sigma, n}(T)$.

7. If $\left\|u_{n}-u_{n-1}\right\|_{V} \leq \varepsilon$ stop. Otherwise, consider $\bar{r}_{n+1}$ (given by (51) and go to Step 5.

Starting from the observation associated with the autonomous undisturbed system, the residual $\bar{r}_{0}=0$ represents the difference between this observation and itself. When the system undergoes a disturbance, $\bar{r}_{1}$ measures the difference between the observations associated respectively with the autonomous undisturbed system (normal state) and the disturbed uncontrolled one. Therefore, the role of $u_{1}$ is to reduce the amount $\bar{r}_{1}$ until the condition given in Step 7 of Algorithm 1 is satisfied. Otherwise, we start the procedure by considering $\bar{r}_{n+1}$ for $n \geq 1$. This allows us to calculate a solution $u$ to Problem $(\bar{P})$. Therefore, by inserting this solution into (13), we can directly determine the state $z_{f, u}$ (and hence the associated output $y_{f, u}^{\sigma}$ ) of the system (12). In addition, the state $\widetilde{z}_{f, u}$ of the original system (1) will be obtained thanks to the variable change made in (9). Note that this is the objective of Remark 4. We can return to the original system (without delay), without determining the operator $\Pi$ given in (36).

From Algorithm 1, we see that determining the protector control $u$ solution to Problem $(\widetilde{P})$ is essentially based on the computation of the pseudoinverse $L$ given by (43). Such computation is not simple to perform, given the dependence of the operator $L$ on various operators of the delay system governed by the state equation (27). What we propose in the next section is to exploit the remediability concept and delay otherwise.

4.2. Adapted families of transformations and remediability. This approach consists in finding a control which permits to deviate the trajectory of the spreadable disturbance so that it will not pass through $\sigma$. Taking into account the expression (6) and the variable change (9), the property $\mathcal{P}$ generated by the disturbance $f$ is given by

$$
\mathcal{P} z_{f, 0}(x, t) \Leftrightarrow z_{f, 0}(x, t) \neq z_{0,0}(x, t)-f(x) .
$$

For that purpose, we recall the following definition (Bernoussi and El Jai, 2000; Bernoussi et al., 2001).

Definition 3. Let $w_{f, 0, t}=\left\{x \in \Omega: \mathcal{P} z_{f, 0}(x, t)\right\}$. The trajectory of $\mathcal{P}$ (or trajectory of $f$ ) is

$$
\mathcal{T}_{T}=\bigcup_{t \in I} w_{f, 0, t} .
$$

If the disturbance $f$ is spreadable in the inclusion sense, then $\mathcal{T}_{T}=w_{f, 0, T}$. But if the spreadability of $f$ is in the measure sense, we can introduce the so-called adapted families of transformations to characterize the trajectory of $f$. We have the following definition (Bernoussi, 2007).

Definition 4. We say that a family of transformations $(F(. ; s, t))_{s \geq t}: \Omega \rightarrow \Omega$ is adapted to the evolution of $(S)$ if

$$
w_{f, 0, s}=F\left(w_{f, 0, t} ; s, t\right), \quad t, s \in I: \quad t \leq s .
$$

In order to protect $\sigma$, we propose an approach based on compensating the effects of disturbance $f$ on another subregion $D$ during a certain time $\tau$ properly chosen before $\sigma$ is reached by the spreadable disturbance $f$. This consists in exploiting the delay in another way. Let $\omega$ be the geometrical support of the spatial disturbance $f$ affecting the system at the initial time $(t=0), \sigma$ a subregion such that $\bar{\omega} \cap \bar{\sigma}=\emptyset$. Then the principle of this approach consists in

1. Determining the trajectory $\mathcal{T}_{T}$ of $f$ in the autonomous disturbed case.

2. Choosing a particular zone $D$ in $\mathcal{T}_{\tau}$ where $\left.\tau \in\right] 0, t_{1}[$ and $t_{1}$ is the time when $f$ starts to reach $\sigma$, such that remediability on $D$ implies the protection of $\sigma$.

This can be illustrated by considering the following examples: one-dimensional (Fig. 2) and multidimensional (Fig. 3). For the first case (Fig. 2), the geometrical support of the disturbance can be positioned on the left $\left(\omega_{1}\right)$ or right $\left(\omega_{2}\right)$ to $\sigma$. When $f$ is spreadable and $\sigma$ is vulnerable, the first time $t_{1}$ where the effects of $f$ start to reach $\sigma$ can be computed. Therefore, the protection of $\sigma$ can be performed by a compensating, before the time $t_{1}$, the effects of $f$ in $D_{1}$ and/or in $D_{2}$ located on the trajectory of 
$f$. Generally, the areas $D_{1}$ and $D_{2}$ are already affected by the effects of the spreadable disturbance that starts from $\omega_{1}$ and/or $\omega_{2}$, but $\sigma$ is not reached yet. This justifies the combination of the two principles of remediability and the protector control, and how this is linked to the notion of delay.

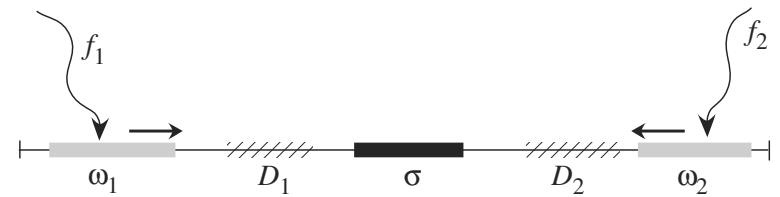

Fig. 2. Spatial localization of the disturbance $\left(\omega_{1}\right.$ and $\left.\omega_{2}\right)$ and the choice of zones $\left(D_{1}\right.$ and/or $\left.D_{2}\right)$.

The second case (Fig. 3) illustrates different situations of the localization zones $D_{k}$ according to the nature of the spreadable phenomenon; in some situations we can consider the case where

$$
D=\bigcup_{k} D_{k}
$$

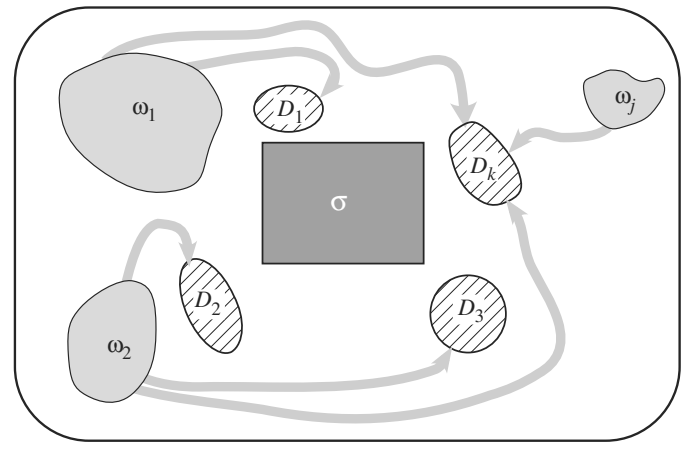

(a)

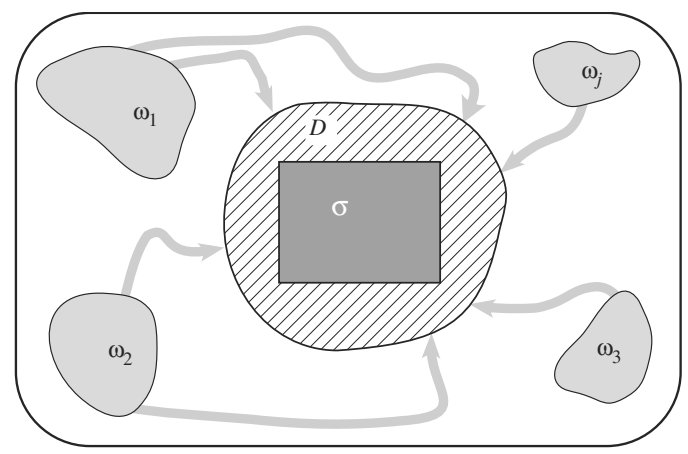

(b)

Fig. 3. Spatial localization of the disturbance $\omega_{j}$ and the choice of zones $D_{k}$

\section{Remark 6.}

1. Qaraai et al. (2008) considered the regional remediability problem on a given zone $D$ for the same class of nonlinear systems.

2. The choice of $D$ as the determination of the adapted family of transformations remains very difficult. However, it is possible, for some class of systems such as transportation ones or those which evolve as a travelling wave, to determine such families (Bernoussi et al., 2001).

Then, based on the principle of this second approach as well as Definitions 1 and 2, the protection of $\sigma$ can be characterized as in the following result.

Proposition 2. Assume that $f$ is spreadable, $\sigma$ is $f$ vulnerable and will be reached at time $t_{1}$ and $D \subset \mathcal{T}_{\tau}$ where $\tau \in] 0, t_{1}[$. If $u$ is an $f$-D-remediable control on $[0, \tau]$ and stuck the disturbance effects in the direction of $\sigma$ (through $D)$, then $\sigma$ is $f$-protectable.

Proof. As $f$ is assumed to be spreadable and $\sigma$ is vulnerable, then the trajectory of $f$ moves in the direction of $\sigma$ and, consequently, it will reach $\sigma$ at the time $t_{1}$ through the zone $D$. As $D$ is assumed to be $f$-remediable during $[0, \tau]$, then there exists a control $u$ which permits to compensate the effects of $f$ on $D$ at time $\tau$. This means that the system find its normal evolution (autonomous undisturbed case) from the time $\tau$. This also means that $\sigma$ would not be vulnerable for every $t \in[\tau, T]$. Taking into account the assumptions on $\tau$ and $t_{1}$, the subregion $\sigma$ would not be vulnerable over the entire time interval $[0, T]$. Consequently, it is $f$-protectable (by Definition 2)

Remark 7. The choice of the time $\tau$ and the subregion $D$ on which we remedy the effects of the disturbance during $[0, \tau]$ depends especially on the spreadability speed of the disturbance $f$ and its trajectory that starts from $\omega$. That is why in this paper we have considered only systems which have a finite propagation velocity.

Thus, from the above, to protect $\sigma$ (e.g., to solve problem $(\widetilde{P})$ ), we have to solve the regional remediability problem on $D$ during $[0, \tau]$ formulated as follows:

$$
\left\{\begin{array}{l}
\text { Find } u \in L^{2}(0, \tau ; \mathcal{U}) \text { such that } \\
\widetilde{y}_{f, u}^{D}(\tau)=\widetilde{y}_{0,0}^{D}(\tau),
\end{array}\right.
$$

where $\widetilde{y}_{f, u}^{D}$ is the output function in the subregion $D$.

\section{Application to bacterial dynamics}

5.1. Discussed model. In this section we consider a variant of Fisher's equation which combines the convection, a logistic term expressed as a quadratic nonlinearity, a growth rate $\rho$ (the difference between the birth and mortality rates) and an environmental parameter $\mu$ (carrying capacity of the environment), see, e.g., the works of Giuggioli and Kenkre (2003) or Kenkre (2004). The diffusive component of the movement is neglected compared with the convective counterpart. This can appear in 
some situations of population dynamics problems, where the transport can lead such a population in one direction, and therefore the effects of convection overwhelm those of diffusion. In the case of the population dynamics of bacteria which can be modelled by such an equation and for certain medical applications, it was shown that, in practice, a moving mask is used to "protect" against bacteria dynamically facing ultraviolet radiation (UV), which kills them in the areas outside the mask (Giuggioli and Kenkre, 2003; Kenkre, 2004).

In our case, we show, through a convective model, how to protect a certain area facing the effects of a given disturbance due to UV radiation using a fixed actuator and a fixed sensor. Note that we designate by the area a subregion containing a certain population density, and by the fixed actuator, an actuator whose support is independent of time (El Jai and Pritchard, 1986).

Let $\Omega=] 0, \pi / 4[, I=] 0, T[$ with $T=2$. We introduce the following notation:

- $\widetilde{z}(x, t)$ the infected population density in $(x, t) \in$ $\Omega \times I$,

- $\widetilde{z}(x, 0)$ the initial infected population density in $x \in$ $\Omega$,

- $\rho(x, t)$ the population growth rate,

- $\mu(x, t)$ the carrying capacity of the environment,

- $f(x)$ the infected population density at initial time $t=0$ in $\omega$ due to UV radiation (disturbance),

- $B u(t)$ the control term,

- $\sigma$ a given subregion in $\Omega$ which contains a healthy population.

At the initial time $\left(t_{0}=0\right)$, and in the absence of UV radiation (e.g., there is no disturbance $f=0$ ), the domain $\Omega$ contains a given number of healthy individuals. Consequently, the initial density of the infected population is zero $\left(\widetilde{z}_{0,0}=0\right)$. But if at the initial time $t_{0}=0$ there is an issue of UV radiation in the region $\omega$, then there will be an infection of the population in such a region. Therefore, if we mean by $f$ the infected population density on $\omega$ which may be likened to a disturbance on the system, and if this disturbance is spreadable, then the healthy individuals become increasingly infected. Note that this infection can be done by a change in the DNA of healthy individuals. Therefore, if the fixed zone $\sigma$ is vulnerable to the effects of $f$, then our goal is the determination of a control $u$ which protects $\sigma$ facing the effects of $f$. To treat this problem, we assume that the quantities $\rho$ and $\mu$ are constant (in space and time), and that the infected density is maintained null at point $x=0$. On $\Omega \times I$, consider the nonlinear convective Fisher equation whose state (e.g., the infected population density) satisfies

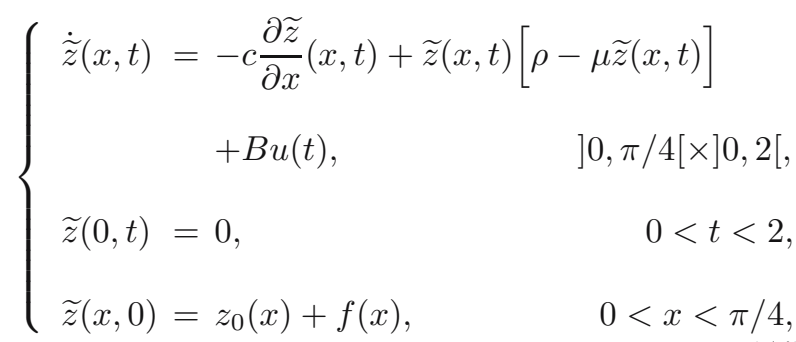

where $\widetilde{z} \in L^{2}(0, T ; Z), Z=L^{2}(\Omega), c>0, \rho>0$ and $\mu>0$.

As an example, we consider $c=3 \pi / 32, \rho=0.5$ and $\mu=0.5$. The initial state $z_{0}$ and the disturbance $f$ are given by

$$
\begin{gathered}
z_{0}(x)=0, \quad \forall x \in \Omega \\
f(x)= \begin{cases}\sin (x), & x \in \omega, \\
0, & \text { elsewhere, }\end{cases}
\end{gathered}
$$

where $\omega=] 0, \pi / 16[$ is the geometrical support of $f$. Let $\sigma$ be a fixed region in $\Omega: \bar{\sigma} \cap \bar{\omega}=\emptyset$, e.g., $\sigma=[3 \pi / 16, \pi / 4]$. The system (54) is augmented by the output function

$$
(\widetilde{E}) \quad \widetilde{y}^{\sigma}(t)=C \chi_{\sigma}^{*} \chi_{\sigma} \widetilde{z}(t)=\int_{0}^{\pi / 4} \chi_{\sigma} \widetilde{z}(x, t) \mathrm{d} x
$$

Consider also the control term $B u(t)=g(x) u(t)$ with

$$
g(x)= \begin{cases}1+\exp \left(x^{2}\right), & 3 \pi / 32 \leq x \leq 5 \pi / 32 \\ 0, & \text { elsewhere }\end{cases}
$$

The system (54) is a particular case of (1) with

$$
F \widetilde{z}=-c \frac{\partial \widetilde{z}}{\partial x}+\widetilde{z}(\rho-\mu \widetilde{z}), \quad B u(t)=g(x) u(t) .
$$

The hypotheses on $F$ are satisfied : $F(0)=0$ and $F$ is $C^{1}$ on $Z$. For all $(x, t) \in \Omega \times I$, consider the change of variables $z(x, t)=\widetilde{z}(x, t)-f(x)$. Then, by linearizing $F$ around $f$, (54) becomes

$$
\left\{\begin{aligned}
\dot{z}(x, t)= & \left(-c \frac{\partial}{\partial x}+\rho-2 \mu f\right) z-\mu z^{2} \\
& +\left(\rho f-\mu f^{2}-c \frac{\partial f}{\partial x}\right)+g(x) u(t), \\
z(0, t)= & 0 \\
z(x, 0)= & 0 .
\end{aligned}\right.
$$


The linear operator

$$
A_{f}=-c \frac{\partial}{\partial x}+\rho-2 \mu f
$$

generates on $Z$ a strongly continuous semigroup given by

$$
\begin{aligned}
& \left(S_{f}(t) z\right)(x) \\
& = \begin{cases}\exp [(\rho-2 \mu f) t] z(x-c t), & x \geq c t \\
0, & 0<x<c t .\end{cases}
\end{aligned}
$$

Then, based on the semigroup approach, the solution $z_{f, u}$ to 55 is given by

$$
\begin{aligned}
& z_{f, u}(x, t) \\
& =-\mu \int_{0}^{t} S_{f}(t-s) z_{f, u}^{2}(x, s) \mathrm{d} s \\
& \quad+\int_{0}^{t} S_{f}(t-s)\left(\rho f-\mu f^{2}-c \frac{\partial f}{\partial x}\right)(x) \mathrm{d} s \\
& \quad+\int_{0}^{t} S_{f}(t-s) g(x) u(s) \mathrm{d} s
\end{aligned}
$$

Moreover, the output 55 is expressed by

$$
\text { (E) } y^{\sigma}(t)=C \chi_{\sigma}^{*} \chi_{\sigma} z(t)=\int_{3 \pi / 16}^{\pi / 4} z(x, t) \mathrm{d} x .
$$

5.2. Numerical simulations. We start by representing the states in the autonomous undisturbed case $(f=0)$ and in the autonomous disturbed one $(f \neq 0)$. This can be represented taking into account of (57) and the change of the variable $z_{f, u}(x, t)=\widetilde{z}_{f, u}(x, t)-f(x)$. We get the results presented in Fig. 4.

In Fig. 4 we represent the state $\widetilde{z}_{0,0}$ of the autonomous undisturbed system (a) and the state of the disturbed uncontrolled system (b). This means that, under the exposure to UV radiation at the initial time, the infected area is increasing.

Consider now the property $\mathcal{P}$ generated by the disturbance $f$ :

$$
\mathcal{P} \widetilde{z}_{f, 0}(x, t) \Leftrightarrow \widetilde{z}_{f, 0}(x, t) \neq \widetilde{z}_{0,0}(x, t),
$$

and besides the subdomains $\left(w_{f, 0, t}\right)_{t}$ and the trajectory $\mathcal{T}_{t}$ of $\mathcal{P}$ (we say also the trajectory of disturbance $f$ ) defined respectively by

$$
w_{f, 0, t}=\left\{x \in \Omega: \mathcal{P} \widetilde{z}_{f, 0}(x, t)\right\} \text { and } \mathcal{T}_{t}=\bigcup_{s \leq t} w_{f, 0, s} .
$$

We remark that the disturbance $f$ is spreadable and the zone $\sigma$ is $f$-vulnerable. Thus, the time $t_{1}$ when $f$ begins to reach $\sigma$ is such that $t_{1}=4 / 3$ (Fig. 5). As

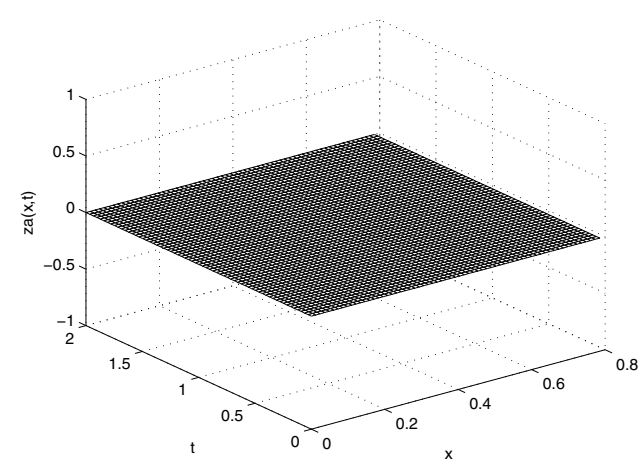

(a)

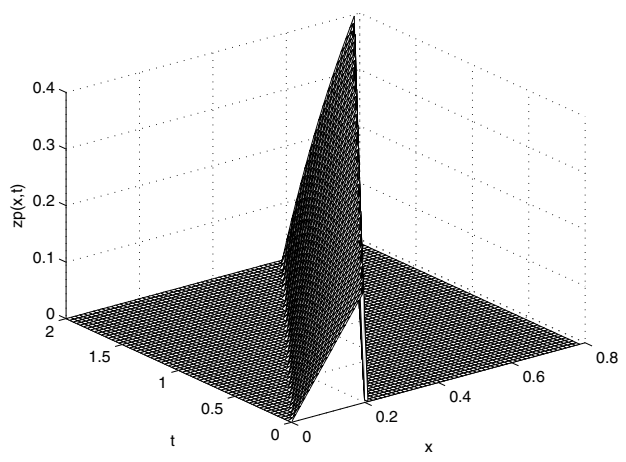

(b)

Fig. 4. States of the autonomous undisturbed system (a) and the autonomous disturbed one (b).

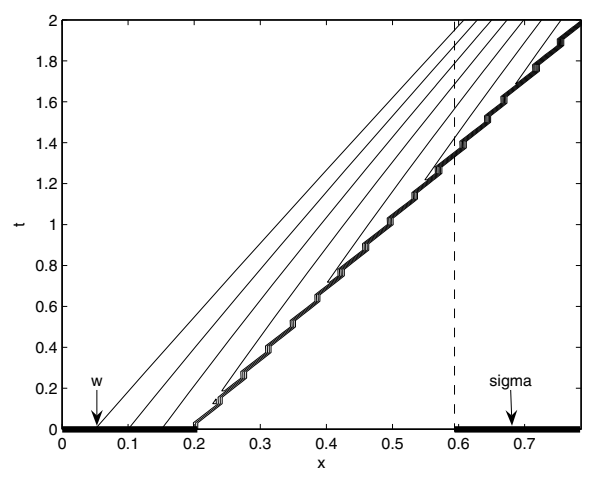

Fig. 5. Trajectory $\mathcal{T}_{t}$ of the disturbance $f$ without the control, and the vulnerability of $\sigma=[3 \pi / 16, \pi / 4] \simeq$ $[0.59,0.78]$

soon as $\sigma$ is reached, we will see how can we protect it with a suitable control $u$ by applying the result of Proposition 2 Therefore, to protect $\sigma$, we can remedy the effects of $f$ on an area $D$ located on the trajectory $\mathcal{T}_{t}$ before 


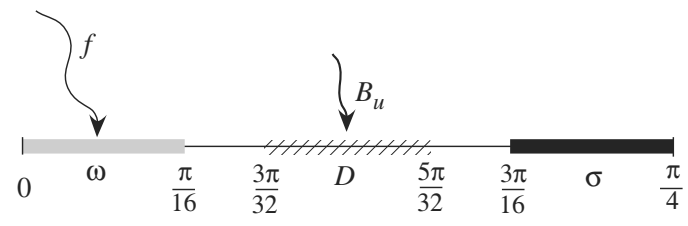

Fig. 6. Different zones considered $(\omega, D$ and $\sigma)$ in $\Omega$.

it passes through $\sigma$. We have for this example: $T=2$, $\mathcal{T}_{T}=\Omega=[0, \pi / 4]$ and $t_{1}=4 / 3, \mathcal{T}_{t_{1}}=[0,3 \pi / 16]$.

Hence, setting $D$ located at $\mathcal{T}_{t_{1}}$, since the trajectory does not reach $\sigma$, the problem of the protector control of $\sigma$ boils down to that of regional remediability on $D$ at a fixed time $\tau$ with $\tau<t_{1}$. Consequently, we have to solve the following remediability problem:

$$
\left\{\begin{array}{l}
\text { Find } u \in L^{2}(0, \tau ; \mathcal{U}) \text { such that } \\
y_{f, u}^{D}(\tau)=y_{0,0}^{D}(\tau)-C \chi_{D}^{*} \chi_{D} f
\end{array}\right.
$$

where $y_{f, u}^{D}$ is the output function on $D$ which is given by

$$
y_{f, u}^{D}(t)=C \chi_{D}^{*} \chi_{D} z_{f, u}(t)=\int_{D} z_{f, u}(x, t) \mathrm{d} x
$$

As $y_{0,0}^{D}(t)=0, \forall t \in I$, it remains to determine a control $u$ such that

$$
\begin{gathered}
-\mu \int_{D} \int_{0}^{\tau} S_{f}(\tau-s) z_{f, u}^{2}(x, s) \mathrm{d} s d x \\
+\int_{D} \int_{0}^{\tau} S_{f}(\tau-s) g(x) u(s) \mathrm{d} s \mathrm{~d} x \\
+\int_{D} \int_{0}^{\tau} S_{f}(\tau-s)\left(\rho f-\mu f^{2}-c \frac{\partial f}{\partial x}\right)(x) \mathrm{d} s \mathrm{~d} x=0 .
\end{gathered}
$$

Consider $D=[3 \pi / 32,5 \pi / 32]$ as shown in Fig. 6, and then the corresponding time $\tau=1$ (e.g., it is the time when the entire zone $D$ is reached by the effets of $f$, see Fig. 5).

The problem (59) can be solved by applying the algorithm developed by Qaraai et al. (2008) to determine a control which permits compensating regionally the effects of the spreadable disturbance.

Remedying the effect of $f$ during the time interval $J=] 0,1[$ on the zone $D=[3 \pi / 32,5 \pi / 32]$, as shown in Fig. 7b), the trajectory of the disturbance $f$ for the disturbed controlled system. We deduce that the control $u$ given in Fig. 8 (b), which compensates $f$ on $D$, enabled stopping the spreadability of $f$ from time $\tau=1$ to the zone $\sigma$. Therefore, it is a $\sigma$-protector control.

Finally, in Fig. 8 (a) we present the corresponding controlled state of the system (54). This shows that, on the zone $\sigma$, the state representing the density of the infected population coincides with the undisturbed autonomous one. Consequently, all individuals occupying the zone $\sigma$ are protected the whole of the time interval $I=] 0,2[$.

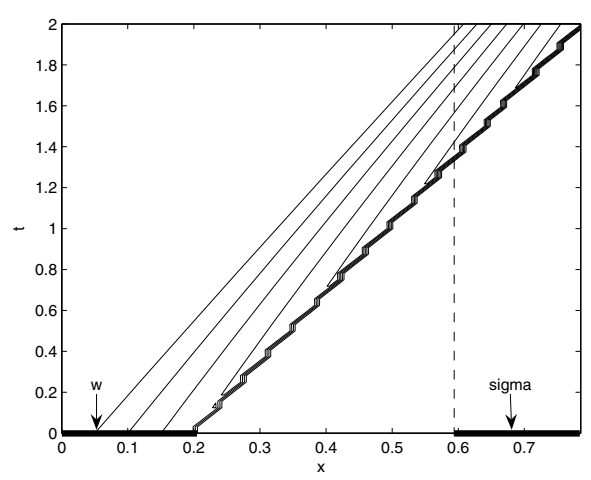

(a)

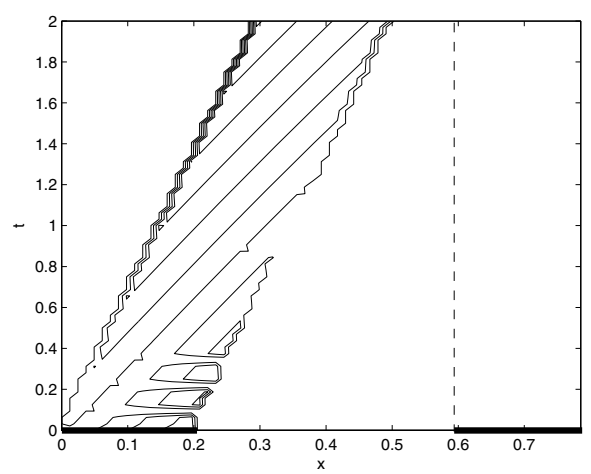

(b)

Fig. 7. Trajectories of $f$ without the control $u$ (a) and in the presence of the control $u$ (b). Under the effect of $u$, $\sigma=[3 \pi / 16, \pi / 4] \simeq[0.59,0.78]$ is $f$-protectable

\section{Conclusion}

In this work, we considered the problem of extending the concept of the protector control, introduced in the linear case (Bernoussi , 2010), to a class of nonlinear distributed parameters systems. This consists in protecting some given areas facing the effect of a disturbance affecting the initial state. To characterize solutions of this problem, a remediability approach for a certain nonlinear delay system was used. Hence, based on pseudoinverse techniques, we solved this problem by giving an algorithm to determine such a control. As was indicated, a difficulty occurred because the direct application of such an algorithm depends on the pseudoinverse operator and various operators of the delay system including nonlinear terms. That is why we considered another approach based on the coupling of families of transformations and a delay. Indeed, given a spreadable disturbance, we showed that it is possible to protect a vulnerable zone by compensating on another affected area located on the trajectory of the disturbance acting before the first time the disturbance begins to reach the area supposed to be protected. This is 


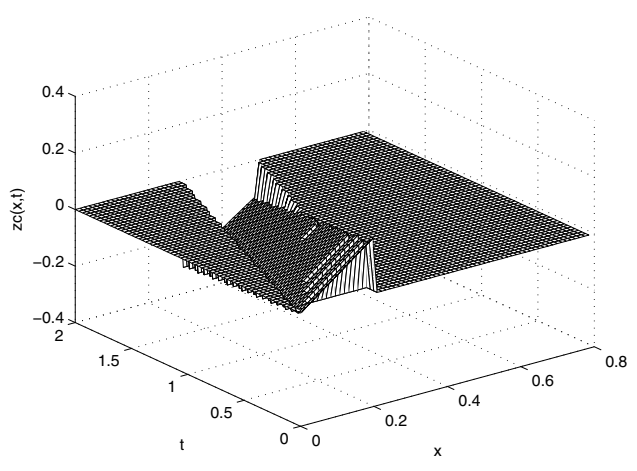

(a)

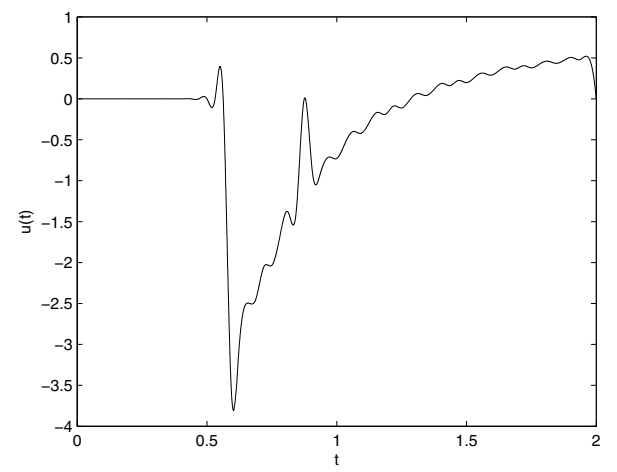

(b)

Fig. 8. State of the disturbed controlled system (54) (a) and the protector control of $\sigma$ (b) at iteration $n=20$.

illustrated by considering a variant of the nonlinear Fisher equation, which can model the problem of bacterial dynamics.

It would be very interesting to implement Algorithm 1 in future works, and, on the other hand, to enlarge the class of systems studied by considering different types of boundary conditions and also when the system does not include only convective terms but also those of diffusion.

\section{Acknowledgment}

This work is supported by the Academy Hassan II of Sciences and Engineering, Morocco.

\section{References}

Afifi, L., Chafiai, A. and El Jai, A. (2002). Regionally efficient and strategic actuators, International Journal of Systems Science 33(4): 1-12.

Afifi, L., El Jai, A. and Merry, M. (2000). Detection and sources reconstruction in a tube, International Journal of Systems Science 31(2): 149-159.
Afifi, L., El Jai, A. and Merry, M. (2001). Regional detection and reconstruction of unknown internal or boundary sources, International Journal of Applied Mathematics and Computer Science 11(2): 319-348.

Beltrami, E. (1987). Mathematics for Dynamic Modelling, Academic Press, San Diego, CA.

Bernoussi, A. (2007). Spreadability and vulnerability of distributed parameter systems, International Journal of Systems Science 38(4): 305-317.

Bernoussi, A. and Amharref, M. (2003). Etalabilitévulnrabilité, Annals of University of Craiova, Mathematics and Computer Science Series 30(4): 53-62.

Bernoussi, A. and El Jai, A. (2000). New approach of spreadability, Journal of Mathematical and Computer Modelling 31(13): 93-109.

Bernoussi, A., El Jai, A. and Pritchard, A. J. (2001). Spreadability and evolving interfaces, International Journal of Systems Science 32(10): 1217-1232.

Bernoussi,A.(2010). Spreadability, vulnerability and protector control, Mathematical Modelling of Natural Phenomena 5(7): 145-150, DOI: 10.1051/mmnp/20105724.

Curtain, R.F. and Pritchard, A.J. (1978). Infinite Dimensional Linear Systems Theory, Springer, Berlin.

Dautray, R.F. and Lions, J.. (1984). Analyse mathématique et calcul numérique pour les sciences et les techniques, Série Scientifique, Tome 3, Masson, Paris.

Diaz, J.I. and Lions, J. L. (1993). Mathematics Climate and Environment, Research in Applied Mathematics, RMA 27, Masson, Paris.

El Jai, A. (2002). Analyse régionale des systémes distribuès, SMAI (Société de Mathématiques Appliquées et Industrielles), ESAIM-European Series in Applied and Industrial Mathematics 8: 663-692.

El Jai, A. (2004). Eléments d'Analyse et Contrôle des Systèmes, Collection études, University of Perpignan Press, Perpignan.

El Jai, A. and Kassara, K. (1994). Spreadable distributed systems, Mathematical and Computer Modelling 20(1): 4764.

El Jai, A. and Kassara, K. (1996). Spreadability of transport systems, International Journal of Systems Science 27(7): 681688.

El Jai, A. and Pritchard, A.J. (1986). Capteurs et actionneurs dans l'analyse des systems distribués, RMA 3, Masson, Paris.

El Jai, A., Simon, M., Zerrik, E. and Pritchard, A.J. (1995). Regional controllability of distributed systems, International Journal of Control 62(6): 1351-1365.

Giuggioli, L. and Kenkre, V.M. (2003). Analytic solutions of a nonlinear convective equation in population dynamics, Physica D 183: 245-259.

Henry, D. (1981). Geometric Theory of Semilinear Parabolic Systems, Lecture Notes in Mathematics, Vol. 840, Springer-Verlag, Berlin/New York, NY. 
Ichikawa, A. (1982). Quadratic control of evolution equations with delays in control, SIAM Journal Control and Optimization 20(5): 645-668.

Kenkre, V.M. (2004). Results from variants of the Fisher equation in the study of epidemics and bacteria, Physica $A$ 342: 242-248.

Pazy, A. (1983). Semigroups of Linear Operators and Applications to Partial Differential Equations, Applied Mathematical Sciences, Vol. 44, Springer-Verlag, New York, NY, Berlin/Heidelberg/Tokyo.

Qaraai, Y. (2008). Compensation des perturbations étalables et contrôle protecteur des zones vulnrables, Ph.D. thesis, Faculty of Sciences and Techniques of Tangier, Morocco.

Qaraai, Y., Bernoussi, A. and El Jai, A. (2006). Contrôle protecteur et remdiabilité, Proceeding of the 3rd International Congress on Scientific Advances in Civil and Industrial Engineering, Algeciras, Spain, pp. 108-114.

Qaraai, Y., Bernoussi, A. and El Jai, A. (2008). How to compensate a spreading disturbance for a class of nonlinear systems, International Journal Applied Mathematics and Computer Science 18(2): 171-187, DOI: 10.2478/v10006008-0016-9.

Qaraai, Y., Bernoussi, A. and El Jai, A. (2009). Protector control for a class of nonlinear systems, Proceeding of the International Conference on Systems Theory: Modeling, Analysis and Control, Fes2009, Fes, Morocco, pp. 265-272.

Smart, D.R. (1994). Fixed Point Theorems, Cambridge University Press, London.
Zerrik, E., El Jai, A. and Amouroux, M. (1994). Regional observability of distributed systems, International Journal of Systems Science 25(6): 301-313.

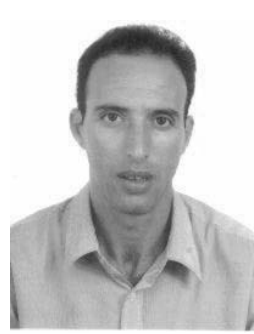

Youssef Qaraai, born in Errachidia, Moroccom (1978), received his Ph.D. degree in applied mathematics (the analysis and control of systems) in 2008, under joint supervision of the Faculty of Sciences and Techniques, Tangier, Morocco, and the University of Perpignan, France. He has a temporary contract at the Department of Mathematics, FST of Tangier (2008-2010), where he continues his post-doctoral research in systems theory with some applications to environment problems and population dynamics.

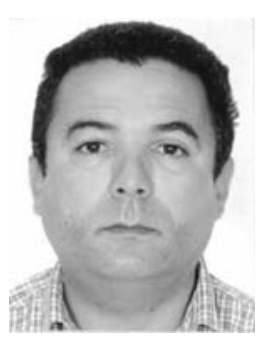

Abdes Samed Bernoussi, born in 1964 (Morocco), received his $\mathrm{Ph} . \mathrm{D}$. degree in applied mathematics (robust control) in 1993 and D.Sc. in systems theory in 2001, from the University of Perpignan, France. He has been the head of the Department of Mathematics at the Faculty of Sciences and Techniques, Tangier, Morocco. His research is related to systems theory and, particularly, the analysis and control of distributed parameters systems: robust control, population dynamics, regional analysis, spreadability, vulnerability. He has published several papers and participated in many conferences and research projects.

Received: 8 July 2009

Revised: 24 March 2010 